\title{
Duality of Floating and Illumination Bodies*
}

\author{
Olaf Mordhorst ${ }^{\dagger}$ and Elisabeth M. Werner ${ }^{\ddagger}$
}

\begin{abstract}
We investigate a duality relation between floating and illumination bodies. The definitions of these two bodies suggest that the polar of the floating body should be similar to the illumination body of the polar. We consider this question for the class of centrally symmetric convex bodies. We provide precise estimates for $B_{p}^{n}$ and for centrally symmetric convex bodies with everywhere positive Gauss curvature. Our estimates show that equality of the polar of the floating body and the illumination body of the polar can only be achieved in the case of ellipsoids.
\end{abstract}

\section{Introduction}

Floating bodies and illumination bodies are attracting considerable interest as their important properties make them effective and powerful tools. Therefore they, and the related affine surface areas, are omnipresent in geometry, e.g., [15, 16, 25, 26, 28, 17, 30, 44, 57] and find applications in many other areas such as information theory, e.g., 2, 32, 56, the study of polytopes and approximation by polytopes [3, 8, 9, 14, 23, 36, 37, 39, 40, 43, and partial differential equations (e.g., [27, 51] and the solutions for the affine Bernstein and Plateau problems by Trudinger and Wang [48, 49, 50]).

Very recent developments are the introduction of the floating body in spherical space [6] and in hyperbolic space [7. This has already given rise to applications in approximation of spherical and hyperbolic convex bodies by polytopes [5] .

A notion of floating body appeared already in the work of C. Dupin [12 in 1822. In 1990, a new definition was given by Schütt and Werner [41] and independently by Bárány and Larman [4]. They introduced the convex floating body as the intersection of all halfspaces whose hyperplanes cut off a set of fixed volume of a convex body (a compact convex set). In contrast to the original definition, the convex floating body is always convex and coincides with Dupin's floating body if it exists.

The illumination body was introduced in [53] as the set of those points whose convex hull with a given convex body have fixed volume.

The definitions of the floating body and the illumination body suggest a possible duality relation, namely that the polar of a floating body of a convex body $K$ is "close" to an illumination body of the polar of $K$. In fact, for the Euclidean unit ball $B_{2}^{n}$, equality can always be achieved. Note however that equality cannot be achieved in general since it was shown in [41] that floating bodies are always strictly convex, but the illumination body of a polytope is always a polytope.

In this paper we clarify the duality relation between floating body and illumination in the case of centrally symmetric convex bodies with $C^{2}$-boundary. We provide asymptotically precise formulas for bodies with everywhere strictly positive Gauss curvature and for $B_{p}^{n}, 2 \leq p<\infty$.

Floating bodies and illumination bodies allow to establish the long sought extensions of an important affine invariant, the affine surface area, to general convex bodies in all dimensions. This was

\footnotetext{
*Keywords:floating bodies, illumination bodies. 2010 Mathematics Subject Classification: 52A....

${ }^{\dagger}$ Partially supported by the German Academic Exchange Service

${ }^{\ddagger}$ Partially supported by NSF grant DMS-1504701
} 
carried out in 41, respectively 53. In both instances, affine surface area appears as a limit of the volume difference of the convex body and its floating body, respectively illumination body. Other extensions - all coincide - were given by Lutwak [28] and Leichtweiss [22].

Here we carry out a limit procedure which leads to a new affine invariant that is different from the affine surface area. It is related to the cone measure of the convex body. These measures play a central role in many aspects of convex geometry, e.g., [10, 11, 31, 32.

In a forthcoming paper we investigate the case of centrally symmetric polytopes which leads to discrete versions of the formulas we derive in the following.

The paper is organized as follows. In the next subsection we introduce notation that is used throughout the paper and present the main theorems and some consequences, a characterization of ellipsoids among them. Section 2 provides the necessary definitions and background. In Section 3 we give an upper bound for general centrally symmetric convex bodies that are $C^{2}$ and in Section 4 we prove that this upper bound is precise in the case of $C_{+}^{2}$-boundary and for $B_{p}^{n}, 2 \leq p<\infty$. We also provide a lower bound for the case $1<p<2$.

\subsection{Notation}

We denote by $\mathbb{R}_{\geq 0}$ the non-negative real numbers. A convex body $K \subseteq$ in $\mathbb{R}^{n}$ is a convex, compact subset of $\mathbb{R}^{n}$ with non-empty interior. $K$ is called centrally symmetric (with respect to the origin) if $K=-K$. From now on, we will always denote by $C \subseteq \mathbb{R}^{n}$ a centrally symmetric convex body and by $S \subseteq \mathbb{R}^{n}$ a centrally symmetric convex body with $C^{2}$-boundary. We refer to the books by Schneider [38] or Gardner [13] for background on convex bodies.

Let $A$ and $B$ be subsets of $\mathbb{R}^{n}$. Then $\operatorname{conv}[A, B]=\{\lambda a+(1-\lambda) b: a \in A, b \in B, 0 \leq \lambda \leq 1\}$ is the convex hull of $A$ and $B$. If $B=\{x\}$, we simply write $\operatorname{conv}[A, x]$. For a measurable set $A \in \mathbb{R}^{n}$, we denote by $|A|_{k}$ its $k$-dimensional Hausdorff measure and, in particular, by $|A|_{n}$ its $n$-dimensional volume.

For $1 \leq p<\infty$, let $B_{p}^{n}$ be the unit ball of the space $\mathbb{R}^{n}$ equipped with the norm $\|x\|_{p}=$ $\left(\sum_{i=1}^{n}\left|x_{i}\right|^{p}\right)^{\frac{1}{p}}$

$$
B_{p}^{n}=\left\{x \in \mathbb{R}^{n}:\|x\|_{p} \leq 1\right\} .
$$

\subsection{Statement of principal results}

Let $K$ be convex body in $\mathbb{R}^{n}$ and $\delta \geq 0$. The convex floating body $K_{\delta}$ of $K$ was introduced in 41 and independently by Bárány and Larman [4] as the intersection of all half spaces whose defining hyperplanes cut off a set of volume $\delta|K|_{n}$ from $K$. More precisely,

$$
K_{\delta}=\bigcap_{\left|K \cap H^{-}\right|_{n} \leq \delta|K|_{n}} H^{+},
$$

where $H$ is a hyperplane and $H^{+}, H^{-}$are the corresponding closed halfspaces. An important result by Meyer and Reisner [29] which we will use throughout, states that for centrally symmetric convex bodies Dupin's floating body always exists and coincides with the convex floating body.

The illumination body $K^{\delta}$ of $K$ was introduced in 53 as follows

$$
K^{\delta}=\left\{x \in \mathbb{R}^{n}:|\operatorname{conv}[K, x]|_{n} \leq(1+\delta)|K|_{n}\right\} .
$$

Note that the illumination body is always convex. This can easily be seen by the fact that

$$
|\operatorname{conv}[K, x]|_{n}=\frac{1}{2}\left(|K|_{n}+\frac{1}{n} \int_{\partial K}|\langle x-y, u(y)\rangle| \mathrm{d} \mu(y)\right),
$$


where $\langle\cdot, \cdot\rangle$ is the standard inner product on $\mathbb{R}^{n}, \mu$ is the surface measure on $\partial K$, the boundary of $K$, and $u(y)$ the almost everywhere uniquely determined outer normal at $y \in \partial K$.

The definitions of the floating body and the illumination body suggest a duality relation, namely that the polar of a floating body of a convex body $K$ is "close" to the illumination body of the polar of $K$,

$$
\left[K_{\delta}\right]^{\circ} \approx\left[K^{\circ}\right]^{\delta^{\prime}}
$$

for suitable $\delta$ and $\delta^{\prime}$. Note that equality cannot be achieved in general since it was shown in [41] that floating bodies are always strictly convex, but the illumination body of a polytope is again a polytope. On the other hand, equality can always be achieved for the Euclidean unit ball $B_{2}^{n}$. We make (1.3) precise in the case of centrally symmetric convex bodies. To do so, we use the distance which we introduce next.

For a convex body $K \subseteq \mathbb{R}^{n}$ with $0 \in K$ and $x \in \mathbb{R}^{n} \backslash\{0\}$ we denote by $r_{K}(x)=\max \{\lambda \geq 0: \lambda x \in$ $K\}$ the radial function of $K$. We define a distance d on the set of $n$-dimensional convex bodies which compares radial functions. We only consider this distance for centrally symmetric convex bodies. If $C_{1}$ and $C_{2}$ are $n$-dimensional centrally symmetric convex bodies, we define We define

$$
\mathrm{d}\left(C_{1}, C_{2}\right)=\sup _{u \in \mathbb{S}^{n-1}} \max \left[\frac{r_{C_{1}}(u)}{r_{C_{2}}(u)}, \frac{r_{C_{2}}(u)}{r_{C_{1}}(u)}\right]=\inf \left\{a \geq 1: \frac{1}{a} C_{1} \subseteq C_{2} \subseteq a C_{1}\right\} \quad .
$$

Note that $\log d(\cdot, \cdot)$ is a metric which induces the same topology as the Hausdorff distance. We put

$$
\langle C\rangle^{\delta}=\left(\left[C^{\circ}\right]^{\delta}\right)^{\circ}
$$

Definition 1.1 Let $C$ be a centrally symmetric convex body and $0<\delta<\frac{1}{2}$. We define

$$
\mathrm{d}_{C}(\delta)=\inf _{\delta^{\prime}>0} \mathrm{~d}\left(C_{\delta},\langle C\rangle^{\delta^{\prime}}\right)
$$

If $C=B_{p}^{n}$, we put $\mathrm{d}_{p}(\delta)=\mathrm{d}_{B_{p}^{n}}(\delta)$.

Please note that $\mathrm{d}_{L(C)}(\delta)=\mathrm{d}_{C}(\delta)$ for every linear invertible map $L$ and that $\mathrm{d}_{2}(\delta)=\mathrm{d}_{B_{2}^{n}}(\delta)=1$.

Together with the distance the distance d, the following expressions will be crucial to make the relation (1.3) precise. For a centrally symmetric convex body $C$ and $x \in \partial C$ with a unique outer normal $u(x)$ and such that the Gauss curvature $\kappa(x)$ exists, we set

$$
G_{C}(x)=c(C, n) \frac{\kappa(x)^{\frac{1}{n+1}}}{\langle x, u(x)\rangle}
$$

where $c(C, n)=\frac{(n+1)^{\frac{2}{n+1}}}{2}\left(\frac{|C|_{n}}{\left|B_{2}^{n-1}\right|_{n-1}}\right)^{\frac{2}{n+1}}$. In most cases we omit the subscript $C$ in $G_{C}$.

Our two main theorems treat the case that the centrally symmetric convex body has $C^{2}$-boundary. If in addition the Gauss curvature is strictly positive everywhere, we say that a convex body is of class $C_{+}^{2}$. For such bodies the function $G: \partial S \rightarrow \mathbb{R}_{\geq 0}$ is continuous with respect to the Euclidean distance. We put

$$
G_{\max }=\max _{x \in \partial S} G(x) \quad \text { and } \quad G_{\min }=\min _{x \in \partial S} G(x) .
$$


Theorem 1.1 Let $S \subset \mathbb{R}^{n}$ be a centrally symmetric convex body that is of class $C^{2}$. Then

$$
\limsup _{\delta \rightarrow 0} \frac{\mathrm{d}_{S}(\delta)-1}{\delta^{\frac{2}{n+1}}} \leq G_{\max }-G_{\min }
$$

Theorem 1.2 Let $S \subset \mathbb{R}^{n}$ be a centrally symmetric convex body that is of class $C^{2}$. If $S$ has everywhere strictly positive Gauss curvature or is an $B_{p}^{n}$-ball, $2 \leq p<\infty$, then

$$
\lim _{\delta \rightarrow 0} \frac{\mathrm{d}_{S}(\delta)-1}{\delta^{\frac{2}{n+1}}}=G_{\max }-G_{\min }
$$

One might ask if Theorem 1.2 holds for general $S$ with $C^{2}$-boundary. However, the authors think that this is not the case and that they can construct a counterexample.

To put the above theorems into context, we recall that it was shown in 41] that for all convex bodies $K$,

$$
\frac{1}{c_{n}} \lim _{\delta \rightarrow 0} \frac{|K|_{n}-\mid K_{\delta /|K|_{n} \mid n}}{\delta^{\frac{2}{n+1}}}=\int_{\partial K} \kappa^{\frac{1}{n+1}}(x) \mathrm{d} \mu(x),
$$

where $c_{n}=\frac{1}{2}\left(\frac{n+1}{\left|B_{2}^{n-1}\right|_{n-1}}\right)^{\frac{2}{n+1}}$ and the right-hand side integral is the well-known affine surface area. Note that a different normalization is chosen for the parameter $\delta$ in the definition of the floating body in 41 .

For $x \in \partial K$ with outer normal $u(x)$, let $m_{K}(x)=\frac{1}{n}\langle x, u(x)\rangle$ be the density function of the cone measure $M_{K}$ of $K$ with respect to the surface measure of $K$. For a Borel set $A \in \partial K$, it is defined as $M_{K}(A)=|\operatorname{conv}[0, A]|_{n}$. We write $n_{K}(x)=\frac{1}{n|K|_{n}}\langle x, u(x)\rangle$ for the density of the normalized cone measure $\mathbb{P}_{K}$ of $K$. This means that

$$
d M_{K}(x)=m_{K}(x) d \mu_{K}(x) \text { and } \quad d \mathbb{P}_{K}(x)=n_{K}(x) d \mu_{K}(x) .
$$

If we rewrite 1.6 using the cone measure equivalently in such a way that have both sides are affine invariants, we get

$$
\lim _{\delta \rightarrow 0} \frac{|K|_{n}-\left|K_{\delta}\right|_{n}}{n|K|_{n} \delta^{\frac{2}{n+1}}}=\int_{\partial K} G(x) \mathrm{d} n_{K}(x)=\|G\|_{1, n_{s}} .
$$

One can consider other differences than the volume difference on the left-hand side of (1.7). Indeed, combining Proposition 3.2 and Lemma 4.3 of the following sections we get a radial-version of (1.7) for centrally symmetric $C^{2}$-bodies

$$
\lim _{\delta \rightarrow 0} \sup _{u \in \mathbb{S}^{n-1}} \frac{r_{S}(u)-r_{S_{\delta}}(u)}{r_{S}(u) \delta^{\frac{2}{n+1}}}=\lim _{\delta \rightarrow 0} \frac{\mathrm{d}\left(S_{\delta}, S\right)}{\delta^{\frac{2}{n+1}}}=G_{\max }=\|G\|_{\infty, n_{s}} .
$$

Let $S$ be a centrally symmetric convex body that is $C_{+}^{2}$. There is a nice way to write $G_{S}$ of 1.5 in terms of cone measures. Let $m_{S}$ and $n_{s}$ be the cone measure respectively the normalized cone measure of $S$ defined above and let $c_{n}$ be as above. Observe that

$$
\begin{aligned}
G_{S}(x) & =c_{n}|S|_{n}^{\frac{2}{n+1}} \frac{\kappa(x)^{\frac{1}{n+1}}}{\langle x, u(x)\rangle}=c_{n}|S|_{n}^{\frac{2}{n+1}}\left(\frac{\kappa(x)}{\langle x, u(x)\rangle^{n}}\right)^{\frac{1}{n+1}}\left(\frac{1}{\langle x, u(x)\rangle}\right)^{\frac{1}{n+1}} \\
& =c_{n}|S|_{n}^{\frac{2}{n+1}}\left(\frac{m_{S^{\circ}}(x)}{m_{S}(x)}\right)^{\frac{1}{n+1}}=c_{n}\left(|S|_{n}\left|S^{\circ}\right|_{n}\right)^{\frac{1}{n+1}}\left(\frac{n_{S^{\circ}}(x)}{n_{S}(x)}\right)^{\frac{1}{n+1}},
\end{aligned}
$$


where $m_{S^{\circ}}$ and $n_{S^{\circ}}$ are defined as follows. Denote by $u_{S}: \partial S \rightarrow \mathbb{S}^{n-1}, x \rightarrow u(x)$ the Gauss map of $S$. Then, $m_{S^{\circ}}(x)=\frac{1}{n} \frac{\kappa_{S}(x)}{\langle x, u(x)\rangle^{n}}$ is the density function of the "cone measure" $M_{S^{\circ}}$ of $S^{\circ}$. For a Borel set $A \in \partial S, M_{S^{\circ}}(A)=\left|\operatorname{conv}\left[0, u_{S^{\circ}}^{-1}\left(u_{S}(A)\right)\right]\right|_{n}$ and $n_{S^{\circ}}(x)=\frac{1}{n\left|S^{\circ}\right|_{n}} \frac{\kappa_{S}(x)}{\langle x, u(x)\rangle^{n}}$ is the density of the normalized cone measure $\mathbb{P}_{S^{\circ}}$ of $S^{\circ}$ (see e.g. [32]). This means that

$$
d M_{S^{\circ}}(x)=m_{S^{\circ}}(x) d \mu_{S}(x) \text { and } d \mathbb{P}_{S^{\circ}}(x)=n_{S^{\circ}}(x) d \mu_{S}(x) .
$$

Thus, the right-hand side of Theorem 1.2 can be re-written as an expression involving the normalized cone measures

$$
\lim _{\delta \rightarrow 0} \frac{\mathrm{d}_{S}(\delta)-1}{\delta^{\frac{2}{n+1}}}=c_{n}\left(|S|_{n}\left|S^{\circ}\right|_{n}\right)^{\frac{1}{n+1}}\left[\max _{x \in \partial S}\left(\frac{n_{S^{\circ}}(x)}{n_{S}(x)}\right)^{\frac{1}{n+1}}-\min _{x \in \partial S}\left(\frac{n_{S^{\circ}}(x)}{n_{S}(x)}\right)^{\frac{1}{n+1}}\right] .
$$

Theorems 1.1 and 1.2 give rise to a new affine invariant which we will also call $G$. We set

$$
\underline{G}(S)=\liminf _{\delta \rightarrow 0} \frac{\mathrm{d}_{S}(\delta)-1}{\delta^{\frac{2}{n+1}}} \text { and } \bar{G}(S)=\limsup _{\delta \rightarrow 0} \frac{\mathrm{d}_{S}(\delta)-1}{\delta^{\frac{2}{n+1}}}
$$

and we put $G(S)=\underline{G}(S)$, if $\underline{G}(S)=\bar{G}(S)$. By a theorem of Petty $\underline{33}$, a centrally symmetric convex body $S$ with $C^{2}$-boundary is an ellipsoid if and only if there is a constant $\alpha_{S}$ such that $\alpha_{S}=\frac{\kappa(x)^{\frac{1}{n+1}}}{\langle x, u(x)\rangle}$ for every $x \in \partial S$. Therefore, $G(S)=0$ if and only if $S$ is an ellipsoid. An immediate consequence of this fact and Theorem 1.2 is the following corollary.

Corollary 1.3 Let $S \subseteq \mathbb{R}^{n}$ be a centrally symmetric convex body with $C_{+}^{2}$-boundary. Suppose there exists a constant $\delta_{0}$ such that for all $0<\delta<\delta_{0}$ and all $\delta^{\prime}>0$ we have that

$$
S_{\delta}=\langle S\rangle^{\delta^{\prime}}
$$

Then $S$ is an ellipsoid.

This corollary supports the conjecture that equality of the floating body of $S$ and the polar of the illumination body of the polar $S^{\circ}$ characterizes ellipsoids. Note that in [42, 47, 57, similar theorems for the homothety conjecture also make use of Petty's lemma as a crucial step in their proofs.

\section{Background}

Let $K \subseteq \mathbb{R}^{n}$ be a convex body and let $x \in \partial K$. If $x$ has a unique outer normal, we denote it by $u_{K}(x)$. We omit the subscript $K$, if it is clear what is meant from the context. If the outer normal is well-defined everywhere, we also denote by $u: \partial K \rightarrow \mathbb{S}^{n-1}, x \rightarrow u(x)$, the Gauss map.

Let $u(x)$ be defined, let $L \in O(n)$ be a rotation such that $L(u(x))=-e_{n}$ and put $K^{\prime}=L(K-x)$. Then there is $\tau>0$ and a convex function $f_{x}: \tau B_{2}^{n-1} \rightarrow \mathbb{R}_{\geq 0}$ with $f(0)=0$ such that the boundary of $K^{\prime}$ is locally around the origin given by the graph of $f_{x}$. We call $f_{x}$ a parametrization of $K$ at $x$. We say that $y \in \partial K$ corresponds to $\left(z, f_{x}(z)\right), z \in \tau B_{2}^{n-1}$, or vice versa, $\left(z, f_{x}(z)\right)$ corresponds to $y$ if $L(y-x)=\left(z, f_{x}(z)\right)$.

If $K$ is differentiable at 0 , then $\nabla f_{x}(0)=0$. If $f_{x}$ is $C^{2}$ on a neighbourhood of 0 , the principal curvatures and the Gauss curvature of $K$ at $x$ are defined as the eigenvalues and the determinant of the Hessian $H f_{x}$ at 0 . We denote the Gauss curvature at $x$ by $\kappa_{K}(x)$ and we omit the subscript $K$ in $\kappa_{K}$ in most cases since the convex body involved will usually be clear from the context. These definitions are independent of the choice of $f_{x}$ (see also the introduction of [34, 43] for the definition of curvature for convex bodies). 
Provided they exist, we denote by $\lambda_{1}(x) \geq \lambda_{2}(x) \geq \cdots \geq \lambda_{n-1}(x) \geq 0$ the principal curvatures at $x \in \partial K$ and we put $\lambda_{\min }=\min _{x \in \partial K} \lambda_{n-1}(x)$ and $\lambda_{\max }=\max _{x \in \partial K} \lambda_{1}(x)$. We put $\kappa_{\min }=$ $\min _{x \in \partial K} \kappa(x)$ and $\kappa_{\max }=\max _{x \in \partial K} \kappa(x)$.

Definition 2.1 Let $K \subseteq \mathbb{R}^{n}$ be a convex body and let $x \in \partial K$ be a boundary point with unique outer normal $u(x)$. We denote by $\Delta_{x}(\delta)>0$ the unique value such that

$$
\left|\left\{y \in K:\langle y, u(x)\rangle \geq\langle x, u(x)\rangle-\Delta_{x}(\delta)\right\}\right|_{n}=\delta|K|_{n} \quad .
$$

Definition 2.2 Let $K \subseteq \mathbb{R}^{n}$ be a convex body with $0 \in \operatorname{int} K$. Let $x \in \partial K$ and $\delta \geq 0$. We denote by $x_{\delta} \in \partial K_{\delta}$ the unique point such that $\left\{x_{\delta}\right\}=K_{\delta} \cap[0, x]$, by $x^{\delta} \in \partial K^{\delta}$ the unique point such that $\{x\}=K \cap\left[0, x^{\delta}\right]$ and by $\langle x\rangle^{\delta} \in \partial\langle K\rangle^{\delta}$ the unique point such that $\left\{\langle x\rangle^{\delta}\right\} \in[0, x] \cap\langle K\rangle^{\delta}$.

We call $y \in \partial K$ a touching point of $x$ with $K$ if the line segment $[y, x]$ lies in a support hyperplane of $K$ at $y$. The following lemma tells us that $\operatorname{conv}[K, x]$ depends only on the touching points of $x$ with $K$. We include proofs of the next two lemmas even though they are probably known.

Lemma 2.1 Let $K \subseteq \mathbb{R}^{n}$ be a convex body and let $x \in \mathbb{R}^{n} \backslash K$. Then

$$
\partial(\operatorname{conv}[K, x]) \subseteq \partial K \cup\{\lambda x+(1-\lambda) y: \lambda \in[0,1], y \in \partial K \text { is a touching point }\} \quad .
$$

Proof. Let $z \in \partial(\operatorname{conv}[K, x])$ and assume that $z \neq x$ and $z \notin \partial K$. It is an elementary fact of convex geometry that $\operatorname{conv}[K, x]=\{\lambda x+(1-\lambda) y: y \in K, \lambda \in[0,1]\}$. A simple consequence is that $\partial(\operatorname{conv}[K, x]) \subseteq\{\lambda x+(1-\lambda) y: y \in \partial K, \lambda \in[0,1]\}$ (see, e.g., [52]). Hence there are $\lambda \in(0,1)$ and $y \in \partial K$ such that $z=\lambda y+(1-\lambda) x$. Let $H$ be a support hyperplane of $\operatorname{conv}[K, x]$ at $z$ and let $H^{+}$ be the corresponding closed halfspace including $\operatorname{conv}[K, x]$. Then $y, x \in H^{+}$, hence, $y, x \in H$ and it follows that $H$ is also a support hyperplane of $\operatorname{conv}[K, x]$ at $y$.

Lemma 2.2 Let $\tau>0$ and let $f:(-\tau, \tau) \rightarrow \mathbb{R}_{\geq 0}$ be convex, of class $C^{2}$ and such that $f(0)=0$, $f^{\prime}(0)=0$ and $f^{\prime \prime}(0)>0$. Let $\frac{1}{16}>\eta \geq 0$. Let $\tau>\theta>0$ be such that $|t| \leq \theta$ implies

$$
\frac{1-\eta}{2} f^{\prime \prime}(0) t^{2} \leq f(t) \leq \frac{1+\eta}{2} f^{\prime \prime}(0) t^{2} .
$$

If $\Delta>0$ is such that $\Delta<\frac{(1-4 \sqrt{\eta}) f^{\prime \prime}(0)}{2} \theta^{2}$, then there exists some $0<t_{0}<\theta$ such that the line through $-\Delta e_{2}$ and $\left(t_{0}, f\left(t_{0}\right)\right)$ is tangent to $f$ at $\left(t_{0}, f\left(t_{0}\right)\right)$. In this case $f\left(t_{0}\right) \leq \frac{1+\eta}{1-4 \sqrt{\eta}} \Delta$.

Proof. For every $s \in(0, \tau)$ the line $l_{s}$ through $-\Delta e_{2}$ and $(s, f(s))$ is given by $l_{s}(t)=\frac{f(s)+\Delta}{s} t-\Delta$ and $l_{s}$ touches the graph of $f$ if and only if $f^{\prime}(s)=\frac{f(s)+\Delta}{s}$. We provide upper and lower estimates for $f^{\prime}(t)$. Put $c=1-2 \sqrt{\eta}$. Since $f$ is convex we have for every $0<t \leq \theta$ that

$$
\begin{aligned}
f^{\prime}(t) & \geq \frac{f(t)-f(t c)}{t-t c} \geq \frac{\frac{1-\eta}{2} f^{\prime \prime}(0) t^{2}-\frac{1+\eta}{2} f^{\prime \prime}(0) c^{2} t^{2}}{(1-c) t}=\left(\frac{1+c}{2}-\eta \frac{1+c^{2}}{2(1-c)}\right) f^{\prime \prime}(0) t \\
& \geq\left(\frac{1+c}{2}-\frac{\eta}{1-c}\right) f^{\prime \prime}(0) t=\left(1-\sqrt{\eta}-\frac{1}{2} \sqrt{\eta}\right) f^{\prime \prime}(0) t=\left(1-\frac{3}{2} \sqrt{\eta}\right) f^{\prime \prime}(0) t
\end{aligned}
$$


Put $d=1+2 \sqrt{\eta}$. Providing that $0<(1+2 \sqrt{\eta}) t \leq \theta$ we get the following upper estimate.

$$
\begin{aligned}
f^{\prime}(t) & \leq \frac{f(d t)-f(t)}{d t-t} \leq \frac{\frac{1+\eta}{2} f^{\prime \prime}(0) d^{2} t^{2}-\frac{1-\eta}{2} f^{\prime \prime}(0) t^{2}}{d t-t}=\left(\frac{1+d}{2}+\eta \frac{1+d^{2}}{2(d-1)}\right) f^{\prime \prime}(0) t \\
& =\left(1+\sqrt{\eta}+\frac{\sqrt{\eta}}{2}+\eta+\eta^{3 / 2}\right) f^{\prime \prime}(0) t \leq\left(1+\frac{7}{2} \sqrt{\eta}\right) f^{\prime \prime}(0) t
\end{aligned}
$$

Put $t=\sqrt{\frac{2 \Delta}{f^{\prime \prime}(0)(1-4 \sqrt{\eta})}}$. Then $t<\theta$ and we get

$$
f^{\prime}(t) t-f(t) \geq\left(1-\frac{3}{2} \sqrt{\eta}\right) f^{\prime \prime}(0) t^{2}-\frac{1+\eta}{2} f^{\prime \prime}(0) t^{2} \geq(1-4 \sqrt{\eta}) \frac{f^{\prime \prime}(0)}{2} t^{2}=\Delta .
$$

Now, put $t=\sqrt{\frac{2 \delta}{f^{\prime \prime}(0)(1+8 \sqrt{\eta})}}$. It is easy to verify that $\frac{t}{1+2 \sqrt{\eta}} \leq \theta$. It follows that

$$
f^{\prime}(t) t-f(t) \leq\left(1+\frac{7}{2} \sqrt{\eta}\right) f^{\prime \prime}(0) t^{2}-\frac{1-\eta}{2} f^{\prime \prime}(0) t^{2} \leq(1+8 \sqrt{\eta}) \frac{f^{\prime \prime}(0)}{2} t^{2}=\Delta \quad
$$

Since $f^{\prime}(t) t-f(t)$ is continuous there is $\sqrt{\frac{2 \delta}{f^{\prime \prime}(0)(1+8 \sqrt{\eta})}} \leq t_{0} \leq \sqrt{\frac{2 \Delta}{f^{\prime \prime}(0)(1-4 \sqrt{\eta})}}$ such that $f^{\prime}\left(t_{0}\right)=$ $\frac{f\left(t_{0}\right)+\Delta}{t_{0}}$. Note that by monotonicity of $f$ we obtain

$$
f\left(t_{0}\right) \leq f\left(\sqrt{\frac{2 \Delta}{f^{\prime \prime}(0)(1-4 \sqrt{\eta})}}\right) \leq \frac{1+\eta}{2} f^{\prime \prime}(0){\sqrt{\frac{2 \Delta}{f^{\prime \prime}(0)(1-4 \sqrt{\eta})}}}^{2} \frac{1+\eta}{1-4 \sqrt{\eta}} \Delta .
$$

We obtain the following immediate generalization for higher dimensions:

Corollary 2.3 Let $\tau>0$ and let $f: \operatorname{int}\left(\tau B_{2}^{n-1}\right) \rightarrow \mathbb{R}_{\geq 0}$ be convex, of class $C^{2}$ such that $f(0)=0$, $\nabla f(0)=0$ and the smallest eigenvalue $\lambda_{n-1}$ of $H f(0)$ is positive. Let $\frac{1}{16}>\eta \geq 0$ and let $\tau>\theta>0$ be such that $\|z\|_{2} \leq \theta$ implies

$$
\frac{1-\eta}{2}\langle H f(0) z, z\rangle \leq f(z) \leq \frac{1+\eta}{2}\langle H f(0) z, z\rangle
$$

If $\Delta>0$ is such that $\Delta<\frac{(1-4 \sqrt{\eta}) \lambda_{n-1}}{2} \theta^{2}$, then for every $v \in \mathbb{S}^{n-2}$ there is some $0<t_{0}(v)<\theta$ such that the line through $-\Delta e_{n}$ and $\left(t_{0}(v) v, f\left(t_{0}(v) v\right)\right)$ lies in the tangent hyperplane of $f$ at $\left(t_{0}(v) v, f\left(t_{0}(v) v\right)\right)$. In this case $f\left(t_{0}(v) v\right) \leq \frac{1+\eta}{1-4 \sqrt{\eta}} \Delta$.

The following lemma can be found in [[35, Lemma 6].

Lemma 2.4 Let $K \subseteq \mathbb{R}^{n}$ a convex body with $C_{+}^{2}$-boundary. There is $\tau>0$ such that for every $\xi \in \partial K$ there is a parametrization $f_{\xi}: \tau B_{2}^{n-1} \rightarrow \mathbb{R}_{\geq 0}$ of $K$ at $\xi$ such that for every $1>\eta>0$ there is $\tau>\theta>0$, independent of $\xi$, such that $\|z\|_{2} \leq \theta$ implies

$$
\frac{1-\eta}{2}\left\langle H f_{\xi}(0) z, z\right\rangle \leq f_{\xi}(z) \leq \frac{1+\eta}{2}\left\langle H f_{\xi}(0) z, z\right\rangle
$$

A careful analysis of the proof in 35] yields the following version of this lemma for $C^{2}$-bodies. 
Lemma 2.5 Let $K \subseteq \mathbb{R}^{n}$ be a convex body with $C^{2}$ boundary. Then there is $\tau>0$ such that for every $\xi \in \partial K$ there is a parametrization $f_{\xi}: \tau B_{2}^{n-1} \rightarrow \mathbb{R}_{\geq 0}$ of $K$ at $\xi$ such that for every $\eta>0$ there is a $\theta$ independent of $\xi, \tau>\theta>0$, such that $\|z\|_{2} \leq \theta$ implies

$$
\frac{1}{2}\left(\left\langle H f_{\xi}(0) y, y\right\rangle-\eta\|y\|^{2}\right) \leq f_{\xi}(z) \leq \frac{1}{2}\left(\left\langle H f_{\xi}(0) y, y\right\rangle+\eta\|y\|^{2}\right) \quad .
$$

As eventually we treat symmetric convex bodies with smooth boundary, we will from now on mostly consider symmetric convex bodies $S$ that are $C^{2}$, even though some of the mentioned results hold true for general convex bodies.

Corollary 2.6 Suppose that $S$ has $C_{+}^{2}$-boundary. For every $\varepsilon>0$ there is $\Delta_{0}>0$ such that for every $0 \leq \Delta \leq \Delta_{0}$ and every $x \in \partial S$ the following holds: Let

$$
S_{1}=S \cap\left\{\xi \in \mathbb{R}^{n}:\langle\xi-x, u(x)\rangle \leq-(1+\varepsilon) \Delta\right\}
$$

and

$$
S_{2}=S \cap\left\{\xi \in \mathbb{R}^{n}:\langle\xi-x, u(x)\rangle \geq-(1+\varepsilon) \Delta\right\} .
$$

Then

$$
\operatorname{conv}[S, x+\Delta u(x)]=S_{1} \cup \operatorname{conv}\left[S_{2}, x+\Delta u(x)\right] .
$$

Proof. Let $\varepsilon>0$ and $\frac{1}{3}>\eta>0$ with $\frac{1+\eta}{1-4 \sqrt{\eta}} \leq 1+\varepsilon$. By Lemma 2.4. we can choose $\tau>0$ and $\tau>\theta>0$ such that for every $x \in \partial S$ the following holds:

There is a parametrization $f_{x}: \tau B_{2}^{n-1} \rightarrow \mathbb{R}_{\geq 0}$ such that

$$
\frac{1-\eta}{2}\left\langle H f_{x}(0) z, z\right\rangle \leq f_{x}(z) \leq \frac{1+\eta}{2}\left\langle H f_{x}(0) z, z\right\rangle,
$$

if $\|z\|_{2} \leq \theta$. Put $\Delta_{0}=\frac{(1-4 \sqrt{\eta}) \lambda_{\min } \theta}{2}$. Let $x_{0} \in \partial S$ and $0<\Delta<\Delta_{0}$. By Lemma 2.1 it is sufficient to show that the touching points of $x_{0}+\Delta u\left(x_{0}\right)$ with $S$ lie in $S_{2}$. If $y \in \partial S$ is a touching point, then there is some $w \in \mathbb{S}^{n-1}$ orthonormal to $u\left(x_{0}\right)$ such that

$$
y \in \partial S \cap\left\{x_{0}+\mu_{1} u\left(x_{0}\right)+\mu_{2} w: \mu_{1}, \mu_{2} \in \mathbb{R}, \mu_{2} \geq 0\right\} \quad .
$$

It is obvious that $y$ is the unique touching point which lies on the halfplane $\left\{x_{0}+\mu_{1} u\left(x_{0}\right)+\mu_{2} w\right.$ : $\left.\mu_{1}, \mu_{2} \in \mathbb{R}, \mu_{2} \geq 0\right\}$. Let $f_{x_{0}}: \tau B_{2}^{n-1} \rightarrow \mathbb{R}_{>0}$ be a parametrization of the boundary at $x_{0}$ and let $v \in \mathbb{S}^{n-2}$ be the vector corresponding to $w$. By Corollary 2.3 there is a $t_{0}(v)$ such that the line through $-\Delta e_{n}$ and $\left(t_{0}(v) v, f_{x_{0}}\left(t_{0}(v)\right)\right)$ is tangential to the graph of $f_{x_{0}}$. Hence, $\left(t_{0}(v) v, f_{x_{0}}\left(t_{0}(v)\right)\right)$ corresponds to the touching point $y$ and $f_{x_{0}}\left(t_{0}(v) v\right) \leq \frac{1+\eta}{1-4 \sqrt{\eta}} \Delta \leq(1+\varepsilon) \Delta$. It follows that

$$
y \in\left\{\xi \in \mathbb{R}^{n}:\left\langle\xi, u\left(x_{0}\right)\right\rangle \geq\left\langle x_{0}, u\left(x_{0}\right)\right\rangle-(1+\varepsilon) \Delta\right\} .
$$

We use Corollary 2.6 to obtain an upper volume estimate for the convex hull of $S$ with a point. Let $\tau=\tau(S)>0$ and $f_{x}: \tau B_{2}^{n-1} \rightarrow \mathbb{R}_{\geq 0}$ be chosen according to Lemma 2.4. We may assume without loss of generality that $\tau$ is chosen so small such that for every $z \in \tau B_{2}^{n-1}$ it holds that $\frac{1}{4}\left\langle H f_{x}(0) z, z\right\rangle \leq f_{x}(z) \leq \frac{3}{4}\left\langle H f_{x}(0) z, z\right\rangle$. For every $v \in \mathbb{S}^{n-2}$,

$$
f_{x}(\tau v) \geq \frac{1}{4}\left\langle H f_{x}(0) \tau v, \tau v\right\rangle \geq \frac{\lambda_{\min } \tau^{2}}{4}=: T_{0}(S) .
$$


Hence, the part of the boundary of $S$ lying in the halfspace $\left\{\xi \in \mathbb{R}^{n}:\langle\xi, u(x)\rangle \geq\langle x, u(x)\rangle-T_{0}(S)\right\}$ is completely parametrized by $f_{x}$, i.e., for every

$$
y \in \partial S \cap\left\{\xi \in \mathbb{R}^{n}:\langle\xi, u(x)\rangle \geq\langle x, u(x)\rangle-T_{0}(S)\right\}
$$

there is a $z \in \tau B_{2}^{n-1}$ such that $\left(z, f_{x}(z)\right)$ corresponds to $y$.

The following two lemmas are uniform versions of well-known estimates for cap and hat volumes which can be found in [21], p. 459] and [53], Lemma 3].

Lemma 2.7 There is a non-negative function $\phi$ with $\lim _{\Delta \rightarrow 0} \phi(\Delta)=0$ such that for every $x \in \partial S$

$$
\frac{(2 \Delta)^{\frac{n+1}{2}}}{n+1} \frac{\left|B_{2}^{n-1}\right|_{n-1}}{(\kappa(x)+\phi(\Delta))^{\frac{1}{2}}} \leq|\{\xi \in S:\langle\xi-x, u(x)\rangle \geq-\Delta\}|_{n} .
$$

Proof. We show that for every $\varepsilon>0$ there is a $\Delta_{0}>0$ such that for every $0 \leq \Delta \leq \Delta_{0}$ we have

$$
\frac{(2 \Delta)^{\frac{n+1}{2}}}{n+1} \frac{\left|B_{2}^{n-1}\right|_{n-1}}{(\kappa(x)+\varepsilon)^{\frac{1}{2}}} \leq|\{\xi \in S:\langle\xi-x, u(x)\rangle \geq-\Delta\}|_{n}
$$

which establishes the proof. Put $\varepsilon^{\prime}=\frac{\varepsilon}{\lambda_{\max } 2^{n-1}}$ and note that $\lambda_{\max }>0$. Assume without loss of generality that $\varepsilon>0$ is chosen sufficiently small so that $\frac{\varepsilon^{\prime}}{\lambda_{\max }} \leq 1$. Apply Lemma 2.5 to $\eta=\frac{\varepsilon^{\prime}}{2}$ and let $\tau>\theta>0$ be chosen accordingly to this $\eta$. Let $H f_{x}(0)=\sum_{i=1}^{n-1} \lambda_{i}(x) v_{i} \otimes v_{i}$. Put $\hat{\lambda}_{i}(x)=\max \left[\lambda_{i}(x), \frac{\varepsilon^{\prime}}{2}\right]$ and $\tilde{H}_{x}=\sum_{i=1}^{n-1} \tilde{\lambda}_{i}(x) v_{i} \otimes v_{i}$. For $w \in \mathbb{S}^{n-2}$ we get

$$
\begin{gathered}
\left\{s \in[-\tau, \tau]: f_{x}(s w) \leq t\right\} \supseteq\left\{s \in[-\theta, \theta]: f_{x}(s w) \leq t\right\} \supseteq\left\{s \in[-\theta, \theta]: \frac{s^{2}}{2}\left(\left\langle H f_{x}(0) w, w\right\rangle+\frac{\varepsilon^{\prime}}{2}\right) \leq t\right\} \\
\supseteq\left\{s \in[-\theta, \theta]: \frac{s^{2}}{2}\left(\left\langle\tilde{H}_{x} w, w\right\rangle+\frac{\varepsilon^{\prime}}{2}\right) \leq t\right\}=\left\{s \in[-\theta, \theta]:|s| \leq \sqrt{\frac{2 t}{\left\langle\tilde{H}_{x} w, w\right\rangle+\frac{\varepsilon}{2}}}\right\} .
\end{gathered}
$$

The second inclusion follows as

$$
f_{x}(s w) \leq \frac{1}{2}\left(\left\langle H f_{x}(0) s w, s w\right\rangle+\frac{\varepsilon^{\prime}}{2}\|s w\|^{2}\right)=\frac{s^{2}}{2}\left(\left\langle H f_{x}(0) w, w\right\rangle+\frac{\varepsilon^{\prime}}{2}\right) .
$$

We may assume that $t \leq \frac{\varepsilon^{\prime}}{4} \theta^{2}$. Note that $s \leq \theta$ since $\left\langle\tilde{H}_{x} w, w\right\rangle+\frac{\varepsilon^{\prime}}{2} \leq \frac{\varepsilon^{\prime}}{2}\|w\|_{2}^{2}+\frac{\varepsilon^{\prime}}{2}=\varepsilon^{\prime}$. This yields

$$
\left\{z \in \tau B_{2}^{n-1}: f_{x}(z) \leq t\right\} \supseteq \sqrt{2 t}\left\{y \in \mathbb{R}^{n-1}:\left\langle\left(\tilde{H}_{x}+\frac{\varepsilon^{\prime}}{2} I\right) y, y\right\rangle \leq 1\right\}
$$

and the set on the right-hand side is an ellipsoid with principle axes $\left(\tilde{\lambda}_{i}(x)+\frac{\varepsilon^{\prime}}{2}\right)^{-1 / 2} v_{i}$. Note that $\left(\tilde{\lambda}_{i}(x)+\frac{\varepsilon^{\prime}}{2}\right)^{-1 / 2} \geq\left(\lambda_{i}(x)+\varepsilon^{\prime}\right)^{-1 / 2}$, which, for $\Delta \leq \frac{\varepsilon^{\prime}}{4} \theta^{2}$, implies that

$$
\begin{aligned}
\int_{0}^{\Delta}\left|\left\{z \in \tau B_{2}^{n-1}: f_{x}(z) \leq t\right\}\right|_{n-1} \mathrm{~d} t & \geq \int_{0}^{\Delta}(2 t)^{\frac{n-1}{2}}\left|\left\{y \in \mathbb{R}^{n-1}:\left\langle\left(\tilde{H}_{x}+\frac{\varepsilon^{\prime}}{2} I\right) y, y\right\rangle \leq 1\right\}\right|_{n-1} \mathrm{~d} t \\
& \geq \frac{(2 \Delta)^{\frac{n+1}{2}}}{n+1} \prod_{i=1}^{n-1} \frac{1}{\left(\lambda_{i}(x)+\varepsilon^{\prime}\right)^{1 / 2}}\left|B_{2}^{n-1}\right|_{n-1}
\end{aligned}
$$


We can conclude, as

$$
\begin{aligned}
\prod_{i=1}^{n-1}\left(\lambda_{i}(x)+\varepsilon^{\prime}\right) & \leq \prod_{i=1}^{n-1} \lambda_{i}(x)+\prod_{i=1}^{n-1}\left(\lambda_{\max }+\varepsilon^{\prime}\right)-\lambda_{\max }^{n-1} \\
& =\kappa(x)+\lambda_{\max }^{n-1}\left(1+\frac{\varepsilon^{\prime}}{\lambda_{\max }}\right)^{n-1}-\lambda_{\max }^{n-1}=\kappa(x)+\lambda_{\max }^{n-1} \sum_{i=1}^{n-1}\left(\begin{array}{c}
n-1 \\
i
\end{array}\right)\left(\frac{\varepsilon^{\prime}}{\lambda_{\max }}\right)^{i} \\
& \leq \kappa(x)+\lambda_{\max }^{n-1} \sum_{i=1}^{n-1}\left(\begin{array}{c}
n-1 \\
i
\end{array}\right) \frac{\varepsilon^{\prime}}{\lambda_{\max }} \leq \kappa(x)+\varepsilon^{\prime} \lambda_{\max }^{n-2} \sum_{i=0}^{n-1}\left(\begin{array}{c}
n-1 \\
i
\end{array}\right) \\
& =\kappa(x)+\varepsilon^{\prime} \lambda_{\max }^{n-2} 2^{n-1}=\kappa(x)+\varepsilon .
\end{aligned}
$$

Lemma 2.8 Suppose that $S$ has $C_{+}^{2}$-boundary. Then there are non-negative functions $\phi$ and $\psi$ with $\lim _{\Delta \rightarrow 0} \phi(\Delta)=0$ and $\lim _{\Delta \rightarrow 0} \psi(\Delta)=0$ such that for every $x \in \partial S$ the following holds.

1.

$$
\frac{(2 \Delta)^{\frac{n+1}{2}}}{n+1} \frac{\left|B_{2}^{n-1}\right|_{n-1}}{\kappa(x)^{\frac{1}{2}}}(1-\phi(\Delta)) \leq|\{\xi \in S:\langle\xi-x, u(x)\rangle \geq-\Delta\}|_{n} \leq \frac{(2 \Delta)^{\frac{n+1}{2}}}{n+1} \frac{\left|B_{2}^{n-1}\right|_{n-1}}{\kappa(x)^{\frac{1}{2}}}(1+\phi(\Delta)) .
$$

2. For every $y \in \mathbb{R}^{n}$ such that $\langle y, u(x)\rangle \geq\langle x, u(x)\rangle+\Delta$ we have

$$
\frac{(2 \Delta)^{\frac{n+1}{2}}}{n(n+1)} \frac{\left|B_{2}^{n-1}\right|_{n-1}}{\kappa(x)^{\frac{1}{2}}}(1-\psi(\Delta)) \leq|\operatorname{conv}[S, y]|_{n}-|S|_{n}
$$

3.

$$
|\operatorname{conv}[S, x+\Delta u(x)]|_{n}-|S|_{n} \leq \frac{(2 \Delta)^{\frac{n+1}{2}}}{n(n+1)} \frac{\left|B_{2}^{n-1}\right|_{n-1}}{\kappa(x)^{\frac{1}{2}}}(1+\psi(\Delta))
$$

Proof. Before we show the volume estimates, we establish some general facts.

Let $0<\varepsilon<1$ and $\Delta_{0}>0$ be chosen according to Corollary 2.6. Let $0<\theta<\tau$ be such that for every $z \in \theta B_{2}^{n-1}$ and every $x \in \partial S$ we have

$$
\frac{1-\varepsilon}{2}\left\langle H f_{x}(0) z, z\right\rangle \leq f_{x}(z) \leq \frac{1+\varepsilon}{2}\left\langle H f_{x}(0) z, z\right\rangle
$$

Without loss of generality we can assume that $(1+\varepsilon) \Delta_{0} \leq T_{0}(S)$ and $\Delta_{0} \leq \frac{(1-\varepsilon) \lambda_{\min } \theta^{2}}{2}$. Let $0 \leq t \leq$ $(1+\varepsilon) \Delta_{0}$. We show that for every $x \in \partial S$,

$$
\sqrt{\frac{2 t}{1+\varepsilon}} \mathcal{E}_{x} \subseteq\left\{z \in \tau B_{2}^{n-1}: f_{x}(z) \leq t\right\} \subseteq \sqrt{\frac{2 t}{1-\varepsilon}} \mathcal{E}_{x}
$$

where $\mathcal{E}_{x}=\left\{\zeta \in \mathbb{R}^{n-1}:\left\langle H f_{x}(0) \zeta, \zeta\right\rangle \leq 1\right\}$ is the indicatrix of Dupin at $x$. For $z \in \tau B_{2}^{n-1} \backslash\{0\}$ it follows for $z \in \theta B_{2}^{n-1}$ that

$$
\begin{aligned}
\frac{(1-\varepsilon) \lambda_{\min }\|z\|_{2}^{2}}{2} & \leq \frac{1-\varepsilon}{2}\left\langle H f_{x}(0) z, z\right\rangle \leq f_{x}(z) \leq \frac{1+\varepsilon}{2}\left\langle H f_{x}(0) z, z\right\rangle \\
& \leq \frac{(1+\varepsilon) \lambda_{\max }\|z\|_{2}^{2}}{2} .
\end{aligned}
$$


Since $\sigma \mapsto f_{x}(\sigma v)$ is strictly monotonously increasing on $[0, \tau]$ for $v \in \mathbb{S}^{n-2}$, we conclude for $\|z\|_{2}>\theta$ and $z^{\prime}=\frac{z}{\|z\|_{2}}$ that

$$
f_{x}(z)>f_{x}\left(\theta z^{\prime}\right) \geq \frac{(1-\varepsilon) \lambda_{\min } \theta^{2}}{2} \geq \Delta_{0} \geq t
$$

Therefore,

$$
\left\{z \in \tau B_{2}^{n-1}: f_{x}(z) \leq t\right\}=\left\{z \in \theta B_{2}^{n-1}: f_{x}(z) \leq t\right\} \subseteq \quad\left\{z \in \theta B_{2}^{n-1}: \frac{1-\varepsilon}{2}\left\langle H f_{x}(0) z, z\right\rangle \leq t\right\} .
$$

Since for $\|z\|_{2}>\theta$ we have $\frac{1-\varepsilon}{2}\left\langle H f_{x}(0) z, z\right\rangle>\frac{(1-\varepsilon) \lambda_{\min } \theta^{2}}{2} \geq t$, it follows that

$$
\left\{z \in \theta B_{2}^{n-1}: \frac{1-\varepsilon}{2}\left\langle H f_{x}(0) z, z\right\rangle \leq t\right\}=\left\{z \in \mathbb{R}^{n-1}: \frac{1-\varepsilon}{2}\left\langle H f_{x}(0) z, z\right\rangle \leq t\right\}=\sqrt{\frac{2 t}{1-\varepsilon}} \mathcal{E}_{x} .
$$

We conclude in a similar way that $\left\{z \in \tau B_{2}^{n-1}: f_{x}(z) \leq t\right\} \supseteq \sqrt{\frac{2 t}{1+\varepsilon}} \mathcal{E}_{x}$.

Proof of 1. Let $x \in \partial S$. Since $\Delta_{0} \leq T_{0}(S)$ the part of the boundary of $S$ lying in the halfspace $\left\{\xi \in \mathbb{R}^{n}:\langle\xi-x, u(x)\rangle \geq-\Delta_{0}\right\}$ is completely parametrized by $f_{x}$. Hence, for every $0 \leq \Delta \leq \Delta_{0}$, the volume of $\{\xi \in S:\langle\xi-x, u(x)\rangle \geq-\Delta\}$ equals the volume of

$$
\left\{(z, t) \in \mathbb{R}^{n}: f_{x}(z) \leq t \leq \Delta\right\} \quad .
$$

Cavalieri's principle and the right-hand side of 2.1 yield

$$
\begin{aligned}
\left|\left\{(z, t) \in \mathbb{R}^{n}: f_{x}(z) \leq t \leq \Delta\right\}\right|_{n} & =\int_{0}^{\Delta}\left|\left\{z \in \tau B_{2}^{n-1}: f_{x}(z) \leq t\right\}\right|_{n-1} \mathrm{~d} t \leq \int_{0}^{\Delta}\left|\sqrt{\frac{2 t}{1-\varepsilon}} \mathcal{E}_{x}\right|_{n-1} \mathrm{~d} t \\
& =\int_{0}^{\Delta}(2 t)^{\frac{n-1}{2}} \mathrm{~d} t \frac{\left|\mathcal{E}_{x}\right|_{n-1}}{(1-\varepsilon)^{\frac{n-1}{2}}}=\frac{(2 \Delta)^{\frac{n+1}{2}}\left|\mathcal{E}_{x}\right|_{n-1}}{n+1}(1-\varepsilon)^{-\frac{n-1}{2}} .
\end{aligned}
$$

Similarly, using the left-hand side of (2.1), one has

$$
\left|\left\{(z, t) \in \mathbb{R}^{n}: f_{x}(z) \leq t \leq \Delta\right\}\right|_{n} \geq \frac{(2 \Delta)^{\frac{n+1}{2}}\left|\mathcal{E}_{x}\right|_{n-1}}{n+1}(1+\varepsilon)^{-\frac{n-1}{2}} .
$$

Since $\operatorname{det}\left(H f_{x}(0)\right)=\kappa(x)$, it follows that $\left|\mathcal{E}_{x}\right|_{n-1}=\kappa(x)^{-1 / 2}\left|B_{2}^{n-1}\right|_{n-1}$. This shows the first part of the lemma.

Proof of 2. Let $0 \leq \Delta \leq \Delta_{0}, y \in\left\{\xi \in \mathbb{R}^{n}\langle\xi-x, u(x)\rangle \geq \Delta\right\}$ and put $B_{x}(\Delta)=\{\xi \in S:\langle\xi-x, u(x)\rangle=$ $-\Delta\}$. The set $\operatorname{conv}[S, y] \backslash S$ includes the set

$$
\operatorname{conv}\left[B_{x}(\Delta), y\right] \backslash\{\xi \in S:\langle\xi-x, u(x)\rangle \geq-\Delta\} \quad
$$

The height of the cone $\operatorname{conv}\left[B_{x}(\Delta), y\right]$ is at least $2 \Delta$. Using Part 1. of the lemma, a lower estimate for the volume of $\operatorname{conv}[S, y] \backslash S$ is

$$
\begin{aligned}
\left|\operatorname{conv}\left[B_{x}(\Delta), y\right]\right|_{n}-|\{\xi \in S:\langle\xi-x, u(x)\rangle \geq-\Delta\}|_{n} \\
\quad \geq \frac{2 \Delta\left|B_{x}(\Delta)\right|_{n-1}}{n}-\frac{(2 \Delta)^{\frac{n+1}{2}}}{n+1} \frac{\left|B_{2}^{n-1}\right|_{n-1}}{\kappa(x)^{\frac{1}{2}}}(1+\phi(\Delta)) .
\end{aligned}
$$

Since $\Delta_{0} \leq T_{0}(S)$, we get similar to the proof of Part 1. that the volume of $\left|B_{x}(\Delta)\right|_{n-1}$ equals $\left|\left\{z \in \tau B_{2}^{n-1}: f_{x}(z) \leq \Delta\right\}\right|_{n-1}$. Using the left-hand side of 2.1 , we obtain

$$
\left|B_{x}(\Delta)\right|_{n-1} \geq \frac{(2 \Delta)^{\frac{n-1}{2}}\left|\mathcal{E}_{x}\right|_{n-1}}{(1+\varepsilon)^{\frac{n-1}{2}}}=\frac{(2 \Delta)^{\frac{n-1}{2}}\left|B_{2}^{n-1}\right|_{n-1}}{\kappa(x)^{1 / 2}}(1+\varepsilon)^{-\frac{n-1}{2}}
$$


which yields

$$
\begin{aligned}
\frac{2 \Delta\left|B_{x}(\Delta)\right|_{n-1}}{n} & -\frac{(2 \Delta)^{\frac{n+1}{2}}}{n+1} \frac{\left|B_{2}^{n-1}\right|_{n-1}}{\kappa(x)^{\frac{1}{2}}}(1+\phi(\Delta)) \\
& \geq \frac{(2 \Delta)^{\frac{2+1}{2}}\left|B_{2}^{n-1}\right|_{n-1}}{\kappa(x)^{1 / 2}}\left(\frac{(1+\varepsilon)^{-\frac{n-1}{2}}}{n}-\frac{1+\phi(\Delta)}{n+1}\right) .
\end{aligned}
$$

The expression $\frac{(1+\varepsilon)^{-\frac{n-1}{2}}}{n}-\frac{1+\phi(\Delta)}{n+1}$ is arbitrarily close to $\frac{1}{n(n+1)}$, if $\Delta_{0}>0$ is small enough to guarantee that $\phi(\Delta)$ is sufficiently small and it is possible to choose $\varepsilon>0$ sufficiently small.

Proof of 3. Let $x \in \partial S$. Since $\Delta_{0}$ is chosen according to Corollary 2.6 we have that for every $0<\Delta \leq \Delta_{0}$ the volume of $\operatorname{conv}[S, x+\Delta u(x)] \backslash S$ is the same as the volume of $\operatorname{conv}\left[S_{2}, x+\Delta u(x)\right] \backslash S_{2}$, where

$$
S_{2}=\{\xi \in S:\langle\xi-x, u(x)\rangle \geq-(1+\varepsilon) \Delta\} .
$$

Since $(1+\varepsilon) \Delta_{0} \leq T_{0}(S)$, the volume of $\operatorname{conv}\left[S_{2}, x+\Delta u(x)\right]$ is given by the volume of $\operatorname{conv}\left[S_{2}^{\prime},-\Delta e_{n}\right]$, where

$$
S_{2}^{\prime}=\left\{(z, t) \in \mathbb{R}^{n-1} \times \mathbb{R}: f_{x}(z) \leq t \leq(1+\varepsilon) \Delta\right\} .
$$

Let $F_{x}(\Delta) \subseteq \mathbb{R}^{n}$ be the cone with base $B_{x}(\Delta)=\sqrt{\frac{\Delta}{2(1-\varepsilon)}}(2+\varepsilon) \mathcal{E}_{x} \times\{(1+\varepsilon) \Delta\}$ and apex $-\Delta e_{n}$, i.e., $F_{x}(\Delta)=\operatorname{conv}\left[B_{x}(\Delta),-\Delta e_{n}\right]$. It follows from the right-hand side of (2.1) that for every $0 \leq t \leq$ $(1+\varepsilon) \Delta$,

$$
\left\{z \in \mathbb{R}^{n-1}:(z, t) \in S_{2}^{\prime}\right\} \subseteq\left\{z \in \mathbb{R}^{n-1}:(z, t) \in F_{x}(\Delta)\right\} .
$$

Thus, $S_{2}^{\prime} \subseteq F_{x}(\Delta)$ and $\operatorname{conv}\left[S_{2}^{\prime},-\Delta e_{n}\right] \subseteq F_{x}(\Delta)$. Since the height of $F_{x}(\Delta)$ is $(2+\varepsilon) \Delta$, the volume of $F_{x}(\Delta)$ is given by

$$
\frac{1}{n}(2+\varepsilon)\left[\sqrt{\frac{2 \Delta}{(1-\varepsilon)}}(2+\varepsilon)\right]^{n-1}\left|\mathcal{E}_{x}\right|_{n-1}=\frac{(2 \Delta)^{\frac{n+1}{2}}\left|B_{2}^{n-1}\right|}{\kappa(x)^{1 / 2}} \frac{\left(1+\frac{\varepsilon}{2}\right)^{n}}{n(1-\varepsilon)^{\frac{n-1}{2}}} .
$$

By Part 1., the volume of $S_{2}$ can be bounded from below by

$$
\frac{(2 \Delta)^{\frac{n+1}{2}}\left|B_{2}^{n-1}\right|_{n-1}}{\kappa(x)^{\frac{1}{2}}} \frac{(1-\phi((1+\varepsilon) \Delta))(1+\varepsilon)^{\frac{n+1}{2}}}{n+1} .
$$

Similar to the proof of Part 2., one can derive the claim of the third part.

\section{Upper Bound}

Let $\Delta_{x}(\delta)$ be as in Definition 2.1

Lemma 3.1 Let $\Gamma_{\min }(\delta)=\min _{x \in \partial S} \frac{\Delta_{x}(\delta)}{\langle x, u(x)\rangle}$ and $\Gamma_{\max }(\delta)=\max _{x \in \partial S} \frac{\Delta_{x}(\delta)}{\langle x, u(x)\rangle}$. Then

$$
\left(1-\Gamma_{\max }(\delta)\right) S \subseteq S_{\delta} \subseteq\left(1-\Gamma_{\min }(\delta)\right) S .
$$

Proof. Let $x \in \partial S$ and $\alpha \geq 0$ such that $0 \in\left\{y \in \mathbb{R}^{n}:\langle y-x, u\rangle \leq-\alpha\right\}$. We show that

$$
\left(1-\frac{\alpha}{\langle x, u\rangle}\right) S \subseteq\left\{y \in \mathbb{R}^{n}:\langle y-x, u\rangle \leq-\alpha\right\} .
$$


Let $\xi \in S$. Then $\langle\xi-x, u\rangle \leq 0$ and $1-\frac{\alpha}{\langle x, u\rangle} \geq 0$, since $0 \in x+\left\{y \in \mathbb{R}^{n}:\langle y, u\rangle \leq-\alpha\right\}$. It follows that

$$
\left\langle\left(1-\frac{\alpha}{\langle x, u\rangle}\right) \xi-x, u\right\rangle=\left(1-\frac{\alpha}{\langle x, u\rangle}\right)\langle\xi-x, u\rangle-\alpha \leq-\alpha
$$

Since

$$
S_{\delta}=\bigcap_{x \in \partial S}\left\{y \in \mathbb{R}^{n}:\langle y-x, u(x)\rangle \leq-\Delta_{x}(\delta)\right\},
$$

the left-hand side inclusion of (3.1) follows immediately from (3.2). For the right-hand side inclusion of (3.1) note that $x_{\delta} \in\left\{\xi \in S:\langle\xi-x, u(x)\rangle \leq-\Delta_{x}(\delta)\right\}$. It follows that

$$
\frac{\left\|x_{\delta}\right\|_{2}}{\|x\|_{2}} \leq \frac{\left\langle x_{\delta}, u(x)\right\rangle}{\langle x, u(x)\rangle}=\frac{\langle x-\Delta(x, \delta) u(x), u(x)\rangle}{\langle x,(u(x))\rangle}=1-\frac{\Delta(x, \delta)}{\langle x, u(x)\rangle} \leq 1-\Gamma_{\min }(\delta) \quad,
$$

i.e. $S_{\delta} \subseteq\left(1-\Gamma_{\min }(\delta)\right) S$

Proposition 3.2 There is a non-negative function $\Phi$ with $\lim _{\delta \rightarrow 0} \Phi(\delta)=0$ such that

$$
\left(1-(1+\Phi(\delta)) G_{\max } \delta^{\frac{2}{n+1}}\right) S \subseteq S_{\delta} .
$$

Proof. We show that for every $\varepsilon>0$ there is a $\delta_{0}>0$ such that for every $0 \leq \delta \leq \delta_{0}$,

$$
\left(1-(1+\varepsilon) G_{\max } \delta^{\frac{2}{n+1}}\right) S \subseteq S_{\delta} .
$$

Let $x_{\max } \in \partial S$ be such that $G\left(x_{\max }\right)=G_{\max }$. Then $\kappa\left(x_{\max }\right)>0$. Put $\mu=\min _{x \in \partial S}\langle x, u(x)\rangle>0$ and $\varepsilon^{\prime}=\frac{\mu^{n+1} \kappa\left(x_{\max }\right) \varepsilon}{\left\langle x_{\max }, u\left(x_{\max }\right)\right\rangle^{n+1}}$. By Lemma 2.7 there is a $\Delta_{0}$ such that for every $0 \leq \Delta \leq \Delta_{0}$ and every $x \in \partial S$,

$$
\frac{(2 \Delta)^{\frac{n+1}{2}}}{n+1} \frac{\left|B_{2}^{n-1}\right|_{n-1}}{\left(\kappa(x)+\varepsilon^{\prime}\right)^{\frac{1}{2}}} \leq|\{\xi \in S:\langle\xi-x, u(x)\rangle \geq-\Delta\}|_{n} .
$$

Choose $\delta_{0}$ such that for every $0 \leq \delta \leq \delta_{0}$ and every $x \in \partial S$ we have that $\Delta_{x}(\delta) \leq \Delta_{0}$. Hence,

$$
\delta|S|_{n} \geq \frac{\left(2 \Delta_{x}(\delta)\right)^{\frac{n+1}{2}}}{n+1} \frac{\left|B_{2}^{n-1}\right|_{n-1}}{\left(\kappa(x)+\varepsilon^{\prime}\right)^{\frac{1}{2}}} .
$$

This yields

$$
\begin{aligned}
\frac{\Delta_{x}(\delta)}{\langle x, u(x)\rangle} & \leq c(S, n) \frac{\left(\kappa(x)+\varepsilon^{\prime}\right)^{\frac{1}{n+1}}}{\langle x, u(x)\rangle} \delta^{\frac{2}{n+1}}=c(S, n)\left(\frac{\kappa(x)}{\langle x, u(x)\rangle^{n+1}}+\frac{\varepsilon^{\prime}}{\langle x, u(x)\rangle^{n+1}}\right)^{\frac{1}{n+1}} \delta^{\frac{2}{n+1}} \\
& \leq c(S, n)\left(\frac{\kappa(x)}{\langle x, u(x)\rangle^{n+1}}+\frac{\varepsilon \kappa\left(x_{\max }\right)}{\left\langle x_{\max }, u\left(x_{\max }\right)\right\rangle^{n+1}}\right)^{\frac{1}{n+1}} \delta^{\frac{2}{n+1}} \\
& \leq c(S, n)\left(\frac{\kappa\left(x_{\max }\right)}{\left\langle x_{\max }, u\left(x_{\max }\right)\right\rangle^{n+1}}+\frac{\varepsilon \kappa\left(x_{\max }\right)}{\left\langle x_{\max }, u\left(x_{\max }\right)\right\rangle^{n+1}}\right)^{\frac{1}{n+1}} \delta^{\frac{2}{n+1}} \\
& =c(S, n) G_{\max }(1+\varepsilon)^{\frac{1}{n+1}} \delta^{\frac{2}{n+1}} \leq c(S, n) G_{\max }(1+\varepsilon) \delta^{\frac{2}{n+1}} .
\end{aligned}
$$

We conclude with Lemma 3.1 
Proposition 3.3 Suppose that $S$ has $C_{+}^{2}$-boundary. Then there is a non-negative function $\Phi$ with $\lim _{\delta \rightarrow 0} \Phi(\delta)=0$ such that

$$
\left(1-(1+\Phi(\delta)) G_{\max } \delta^{\frac{2}{n+1}}\right) S \subseteq S_{\delta} \subseteq\left(1-(1-\Phi(\delta)) G_{\min } \delta^{\frac{2}{n+1}}\right) S .
$$

Proof. Let $1>\varepsilon>0$ and let $\phi$ be the function of Lemma 2.8. Let $\Delta_{0}=\Delta_{0}(\varepsilon)>0$ be sufficiently small, such that for every $0 \leq \Delta \leq \Delta_{0}$ we have $\phi(\Delta) \leq \varepsilon$. Let $\delta_{0}>0$ be such that

$$
\frac{\left(2 \Delta_{0}\right)^{\frac{n+1}{2}}}{n+1} \frac{\left|B_{2}^{n-1}\right|_{n-1}}{\kappa_{\max }^{1 / 2}}(1-\varepsilon)=\delta_{0}|S|_{n}
$$

Let $x \in \partial S$. If $\delta \leq \delta_{0}$, then $\Delta_{x}(\delta) \leq \Delta_{0}$. Indeed, if $\Delta_{x}(\delta)>\Delta_{0}$ then

$$
\begin{aligned}
\left|\left\{\xi \in S:\langle\xi-x, u(x)\rangle \geq-\Delta_{x}(\delta)\right\}\right|_{n} & >\left|\left\{\xi \in S:\langle\xi-x, u(x)\rangle \geq-\Delta_{0}\right\}\right|_{n} \\
& \geq \frac{\left(2 \Delta_{0}\right)^{\frac{n+1}{2}}}{n+1} \frac{\left|B_{2}^{n-1}\right|_{n-1}}{\kappa_{\max }^{1 / 2}}(1-\varepsilon)=\delta_{0}|S|_{n}
\end{aligned}
$$

Since for $\delta \leq \delta_{0}$ we have $\Delta_{x}(\delta) \leq \Delta_{0}$, we obtain the estimate

$$
\frac{G_{\min }}{(1+\varepsilon)^{\frac{2}{n+1}}} \delta^{\frac{2}{n+1}} \leq \frac{c(S, n)}{(1+\varepsilon)^{\frac{2}{n+1}}} \cdot \frac{\kappa(x)^{\frac{1}{n+1}}}{\langle x, u(x)\rangle} \delta^{\frac{2}{n+1}} \leq \frac{\Delta_{x}(\delta)}{\langle x, u(x)\rangle} \leq \frac{c(S, n)}{(1-\varepsilon)^{\frac{2}{n+1}}} \cdot \frac{\kappa(x)^{\frac{1}{n+1}}}{\langle x, u(x)\rangle} \delta^{\frac{2}{n+1}} \leq \frac{G_{\max }}{(1-\varepsilon)^{\frac{2}{n+1}}} \delta^{\frac{2}{n+1}}
$$

Now apply Lemma 3.1 .

Proposition 3.4 Suppose that $S$ has $C_{+}^{2}$-boundary. Then there is a non-negative function $\tilde{\Psi}$ with $\lim _{\delta \rightarrow 0} \tilde{\Psi}(\delta)=0$ such that

$$
\left(1+(1-\tilde{\Psi}(\delta)) n^{\frac{2}{n+1}} G_{\min } \delta^{\frac{2}{n+1}}\right) S \subseteq S^{\delta} \subseteq\left(1+(1+\tilde{\Psi}(\delta)) n^{\frac{2}{n+1}} G_{\max } \delta^{\frac{2}{n+1}}\right) S
$$

Proof. For $x \in \partial S$, let $\Delta^{x}(\delta) \geq 0$ be defined as the value such that

$$
\left|\operatorname{conv}\left[S, x+\Delta^{x}(\delta) u(x)\right]\right|_{n}-|S|_{n}=\delta|S|_{n} \quad .
$$

Let $\varepsilon>0$ be given and let $\Delta_{0}>0$ be such that for every $0 \leq \Delta \leq \Delta_{0}$ the function $\psi$ of Lemma 2.8 is smaller than or equal to $\varepsilon$. We show that there is $\delta_{0}$ such that for every $0 \leq \delta \leq \delta_{0}$ and every $y \in \mathbb{R}^{n}$ with $|\operatorname{conv}[S, y]|_{n}-|S|_{n}=\delta|S|_{n}$, it follows that $\langle y-x, u(x)\rangle \leq \Delta_{0}$ for every $x \in \partial S$. In particular, $\Delta^{x}(\delta) \leq \Delta_{0}$. Let $\delta_{0}>0$ be such that

$$
\frac{\left(2 \Delta_{0}\right)^{\frac{n+1}{2}}}{n(n+1)} \frac{\left|B_{2}^{n-1}\right|_{n-1}}{\kappa_{\max }^{1 / 2}}(1-\varepsilon)=\delta_{0}|S|_{n}
$$

Then arguments similar to the ones in the proof of Proposition 3.3 ensure that $\delta_{0}$ has the desired properties.

Let $\delta \leq \delta_{0}$. We start with the right-hand side inclusion of $\left[3.4\right.$. Since $\left|\operatorname{conv}\left[S, x^{\delta}\right]\right|_{n}-|S|_{n}=\delta|S|_{n}$, it follows that $\left\langle x^{\delta}-x, u(x)\right\rangle=: \Delta \leq \Delta_{0}$. We conclude with Lemma 2.8, 2., that

$$
\delta|S|_{n} \geq \frac{(2 \Delta)^{\frac{n+1}{2}}}{n(n+1)} \frac{\left.B_{2}^{n-1}\right|_{n-1}}{\kappa(x)^{1 / 2}}(1-\varepsilon)
$$


or, equivalently

$$
\Delta \leq \frac{n^{\frac{2}{n+1}} c(S, n)}{(1-\varepsilon)^{\frac{2}{n+1}}} \kappa(x)^{\frac{1}{n+1}} \delta^{\frac{2}{n+1}} .
$$

Hence,

$$
\frac{\left\|x^{\delta}\right\|_{2}}{\|x\|_{2}}=\frac{\left\langle x^{\delta}, u(x)\right\rangle}{\langle x, u(x)\rangle}=1+\frac{\Delta}{\langle x, u(x)\rangle} \leq 1+\frac{n^{\frac{2}{n+1}} G(x)}{(1-\varepsilon)^{\frac{2}{n+1}}} \delta^{\frac{2}{n+1}} \leq 1+\frac{n^{\frac{2}{n+1}} G_{\max }}{(1-\varepsilon)^{\frac{2}{n+1}}} \delta^{\frac{2}{n+1}},
$$

which proves the right-hand side of (3.4).

For the left-hand side inclusion, let again $x \in \partial S$. Then there is $x^{\prime} \in \partial S$ such that $x^{\delta}=x^{\prime}+\Delta^{x^{\prime}}(\delta) u\left(x^{\prime}\right)$, i.e., $x^{\prime}$ is the point of $S$ with minimal distance to $x^{\delta}$. This point is unique since $S$ is strictly convex. Note that $\left\langle x-x^{\prime}, u\left(x^{\prime}\right)\right\rangle \leq 0$, i.e., $\left\langle x, u\left(x^{\prime}\right)\right\rangle \leq\left\langle x^{\prime}, u\left(x^{\prime}\right)\right\rangle$ and therefore,

$$
\frac{\left\|x^{\delta}\right\|_{2}}{\|x\|_{2}}=\frac{\left\langle x^{\delta}, u\left(x^{\prime}\right)\right\rangle}{\left\langle x, u\left(x^{\prime}\right)\right\rangle} \geq \frac{\left\langle x^{\prime}+\Delta^{x^{\prime}}(\delta) u\left(x^{\prime}\right), u\left(x^{\prime}\right)\right\rangle}{\left\langle x^{\prime}, u\left(x^{\prime}\right)\right\rangle}=1+\frac{\Delta^{x^{\prime}}(\delta)}{\left\langle x^{\prime}, u\left(x^{\prime}\right)\right\rangle} .
$$

Since $\Delta^{x^{\prime}}(\delta) \leq \Delta_{0}$, Lemma 2.8 yields

$$
\frac{\Delta^{x^{\prime}}(\delta)}{\left\langle x^{\prime}, u\left(x^{\prime}\right)\right\rangle} \geq \frac{n^{\frac{2}{n+1}} G\left(x^{\prime}\right)}{(1+\varepsilon)^{\frac{2}{n+1}}} \delta^{\frac{2}{n+1}} \geq \frac{n^{\frac{2}{n+1}} G_{\min }}{(1+\varepsilon)^{\frac{2}{n+1}}} \delta^{\frac{2}{n+1}} .
$$

This establishes the left-hand side inclusion of (3.4).

Note that $S^{\circ}$ is also a centrally symmetric convex body with $C_{+}^{2}$-boundary (see [18]). An immediate corollary of Proposition 3.4 is the following corollary.

Corollary 3.5 Suppose that $S$ has $C_{+}^{2}$-boundary. Then there is a non-negative function $\Psi$ with $\lim _{\delta \rightarrow 0} \Psi(\delta)=0$ such that

$$
\left(1-(1+\Psi(\delta)) \frac{\tilde{c}(S, n)}{G_{\min }} \delta^{\frac{2}{n+1}}\right) S \subseteq\langle S\rangle^{\delta} \subseteq\left(1-(1-\Psi(\delta)) \frac{\tilde{c}(S, n)}{G_{\max }} \delta^{\frac{2}{n+1}}\right) S,
$$

where $\tilde{c}(S, n)=n^{\frac{2}{n+1}} c(S, n) c\left(S^{\circ}, n\right)$.

Proof. The corollary follows immediately from the following result which can be found in e.g., [18). For every $x \in \partial S$ there exists a unique $y \in \partial S^{\circ}$ with $\langle x, y\rangle=1$ and in this case

$$
\frac{\kappa_{S}(x)^{\frac{1}{n+1}}}{\left\langle x, u_{S}(x)\right\rangle}=\left[\frac{\kappa_{S^{\circ}}(y)^{\frac{1}{n+1}}}{\left\langle y, u_{S^{\circ}}(y)\right\rangle}\right]^{-1} .
$$

\section{Theorem 3.6}

$$
\limsup _{\delta \rightarrow 0} \frac{\mathrm{d}_{S}(\delta)-1}{\delta^{\frac{2}{n+1}}} \leq G_{\max }-G_{\min }
$$

Proof. First assume that there is $x \in \partial S$ with $\kappa(x)=0$. Thus $G_{\max }-G_{\min }=G_{\max }-0=G_{\max }$. Note that

$$
\mathrm{d}_{S}(\delta)=\inf _{\delta^{\prime} \geq 0} \mathrm{~d}\left(S_{\delta},\langle S\rangle^{\delta^{\prime}}\right) \leq \mathrm{d}\left(S_{\delta},\langle S\rangle^{0}\right)=\mathrm{d}\left(S_{\delta}, S\right) .
$$


By Proposition 3.2. $\left(1-G_{\max } \delta^{\frac{2}{n+1}}(1+o(1))\right) S \subseteq S_{\delta} \subseteq S$ and this yields

$$
\mathrm{d}\left(S_{\delta}, S\right) \leq \frac{1}{1-G_{\max } \delta^{\frac{2}{n+1}}(1+o(1))}=1+G_{\max } \delta^{\frac{2}{n+1}}(1+o(1)) .
$$

Hence,

$$
\limsup _{\delta \rightarrow 0} \frac{\mathrm{d}_{S}(\delta)-1}{\delta^{\frac{2}{n+1}}} \leq \lim _{\delta \rightarrow 0} G_{\max }(1+o(1))=G_{\max } .
$$

Assume now that $S$ has $C_{+}^{2}$-boundary. Put $\delta^{\prime}=\frac{G_{\max } G_{\min }}{\tilde{c}(S, n)} \delta^{\frac{2}{n+1}}$. By Proposition 3.3

$$
\left(1-G_{\max } \delta^{\frac{2}{n+1}}(1+o(1))\right) S \subseteq S_{\delta} \subseteq\left(1-G_{\min } \delta^{\frac{2}{n+1}}(1+o(1))\right) S .
$$

By Corollary 3.5 .

$$
\left(1-G_{\max } \delta^{\frac{2}{n+1}}(1+o(1))\right) S \subseteq\langle S\rangle^{\delta^{\prime}} \subseteq\left(1-G_{\min } \delta^{\frac{2}{n+1}}(1+o(1))\right) S .
$$

Therefore, a sufficient condition for $\frac{1}{a} S_{\delta} \subseteq\langle S\rangle^{\delta^{\prime}}$ is

$$
\frac{1}{a}\left(1-G_{\min } \delta^{\frac{2}{n+1}}(1+o(1))\right) \leq 1-G_{\max } \delta^{\frac{2}{n+1}}(1+o(1))
$$

and a sufficient condition for $\langle S\rangle^{\delta^{\prime}} \subseteq a S_{\delta}$ is

$$
1-G_{\min } \delta^{\frac{2}{n+1}}(1+o(1)) \leq a\left(1-G_{\max } \delta^{\frac{2}{n+1}}(1+o(1))\right) .
$$

These two conditions are met, if one takes $a=1+\left(G_{\max }-G_{\max }\right) \delta^{\frac{2}{n+1}}(1+o(1))$. Hence,

$$
\limsup _{\delta \rightarrow 0} \frac{\mathrm{d}_{S}(\delta)-1}{\delta^{\frac{2}{n+1}}} \leq \lim _{\delta \rightarrow 0} \frac{1+\left(G_{\max }-G_{\min }\right) \delta^{\frac{2}{n+1}}(1+o(1))-1}{\delta^{\frac{2}{n+1}}}=G_{\max }-G_{\min }
$$

\section{Lower Bounds}

We prove $\liminf \inf _{\delta \rightarrow 0} \frac{\mathrm{d}_{S}(\delta)-1}{\delta^{\frac{2}{n+1}}} \geq G_{\max }-G_{\min }$ for $S$ with $C_{+}^{2}$-boundary and for $B_{p}^{n}, 2 \leq p<\infty$. We also provide a lower bound for the case $1<p<2$. Together with Theorem 3.6. we get the following.

Theorem 4.1 Let $S \subseteq \mathbb{R}^{n}$ be of class $C_{+}^{2}$ or $S=B_{p}^{n}, 2 \leq p<\infty$. Then

$$
\lim _{\delta \rightarrow 0} \frac{\mathrm{d}_{S}(\delta)-1}{\delta^{\frac{2}{n+1}}}=G_{\max }-G_{\min }
$$

We need another lemma.

Lemma 4.2 For every $x \in \partial S$ the following holds.

1. There is a function $\Phi_{x}$ with $\lim _{\delta \rightarrow 0} \Phi_{x}(\delta)=0$ such that

$$
\left(1-\left(1+\Phi_{x}(\delta)\right) G(x) \delta^{\frac{2}{n+1}}\right) x \in \partial S_{\delta} .
$$


2. If $S$ has $C_{+}^{2}$-boundary then there is a function $\Psi_{x}$ with $\lim _{\delta \rightarrow 0} \Psi_{x}(\delta)=0$ such that

$$
\left(1-\left(1+\Psi_{x}(\delta)\right) \frac{\tilde{c}(S, n)}{G(x)} \delta^{\frac{2}{n+1}}\right) x \in \partial\left[\langle S\rangle^{\delta}\right],
$$

where $\tilde{c}(S, n)$ is the constant defined in Lemma 3.5 .

In order to prove this lemma, we need two results. The first lemma is an immediate consequence of Lemmas 7 and 10 of 41$]$.

Lemma 4.3 Let $K \subseteq \mathbb{R}^{n}$ be a convex body with 0 in its interior and $x \in \partial K$ such that the Gauss curvature $\kappa(x)$ exists. Then

$$
\lim _{\delta \rightarrow 0} \frac{1}{\delta^{\frac{2}{n+1}}} \cdot \frac{\|x\|_{2}-\left\|x_{\delta}\right\|_{2}}{\|x\|_{2}}=\frac{(n+1)^{\frac{2}{n+1}}}{2}\left(\frac{|K|_{n}}{\left|B_{2}^{n-1}\right|_{n-1}}\right)^{\frac{2}{n+1}} \frac{\kappa(x)^{\frac{1}{n+1}}}{\langle x, u(x)\rangle}=G(x),
$$

where $x_{\delta}$ is the unique point lying in the intersection of $\partial K_{\delta}$ with the line segment $[0, x]$.

The second lemma is a direct consequence of Lemma 3 of [53.

Lemma 4.4 Let $K \subseteq \mathbb{R}^{n}$ be a convex body with 0 in its interior and $x \in \partial K$ such that the Gauss curvature $\kappa(x)$ exists. Then

$$
\lim _{\delta \rightarrow 0} \frac{1}{\delta^{\frac{2}{n+1}}} \cdot \frac{\left\|x^{\delta}\right\|_{2}-\|x\|_{2}}{\|x\|_{2}}=\frac{n^{\frac{2}{n+1}}(n+1)^{\frac{2}{n+1}}}{2}\left(\frac{|K|_{n}}{\left|B_{2}^{n-1}\right|_{n-1}}\right)^{\frac{2}{n+1}} \frac{\kappa(x)^{\frac{1}{n+1}}}{\langle x, u(x)\rangle}=n^{\frac{2}{n+1}} G(x)
$$

where $x^{\delta}$ is the unique point on the boundary of $K^{\delta}$ such that $x$ lies on the line segment $\left[0, x^{\delta}\right]$.

Proof of Lemma 4.2. It follows immediately from Lemma 4.3 that for every $x \in \partial S$ there is a function $\Phi_{x}$ such that $\lim _{\delta \rightarrow 0} \Phi_{x}(\delta)=0$ and

$$
\left(1-\left(1+\Phi_{x}(\delta)\right) G(x) \delta^{\frac{2}{n+1}}\right) x \in \partial S_{\delta}
$$

The polar body $S^{\circ}$ has $C^{2}$-boundary with everywhere positive Gauss curvature (see [18]) and for every $x \in \partial S$ there is a unique dual point $y$, i.e. $y \in \partial S^{\circ}$ such that $\langle x, y\rangle=1$ and

$$
\frac{\kappa(x)^{\frac{1}{n+1}}}{\langle x, u(x)\rangle}=\left[\frac{\kappa(y)^{\frac{1}{n+1}}}{\langle y, u(y)\rangle}\right]^{-1} .
$$

By Lemma 4.4

$$
\lim _{\delta \rightarrow 0} \frac{1}{\delta^{\frac{2}{n+1}}} \frac{\left\|y^{\delta}\right\|-\|y\|}{\|y\|}=n^{\frac{2}{n+1}} c\left(S^{\circ}, n\right) \frac{\kappa(y)^{\frac{1}{n+1}}}{\langle y, u(y)\rangle}=n^{\frac{2}{n+1}} c\left(S^{\circ}, n\right) \frac{\langle x, u(x)\rangle}{\kappa(x)^{\frac{1}{n+1}}}=\frac{\tilde{c}(S, n)}{G(x)} .
$$

It follows that there is a function $\tilde{\Psi}_{y}$ with $\lim _{\delta \rightarrow 0} \tilde{\Psi}_{y}(\delta)=0$ such that

$$
\left(1+\left(1+\tilde{\Psi}_{y}(\delta)\right) \frac{\tilde{c}(S, n)}{G(x)} \delta^{\frac{2}{n+1}}\right) y \in\left(S^{\circ}\right)^{\delta} .
$$


Let $x \in \partial S$ and $y \in \partial S^{\circ}$ with $\langle x, y\rangle=1$. We show that we may choose a function $\Psi_{x}$ such that $\lim _{\delta \rightarrow 0} \Psi_{x}(\delta)=0$ and such that

$$
\left(1-\left(1+\Psi_{x}(\delta)\right) \frac{\tilde{c}(S, n)}{G(x)} \delta^{\frac{2}{n+1}}\right) x \in \partial\left[\left(S^{\circ}\right)^{\delta}\right]^{\circ}=\langle S\rangle^{\delta} .
$$

Let $\lambda(\delta)$ be such that $\lambda(\delta) x \in\langle S\rangle^{\delta}$. Then

$$
1 \geq\left\langle\lambda(\delta) x,\left(1+\left(1+\tilde{\Psi}_{y}(\delta)\right) \frac{\tilde{c}(S, n)}{G(x)} \delta^{\frac{2}{n+1}}\right) y\right\rangle=\lambda(\delta)\left(1+\left(1+\tilde{\Psi}_{y}(\delta)\right) \frac{\tilde{c}(S, n)}{G(x)} \delta^{\frac{2}{n+1}}\right) .
$$

Hence we obtain that $\lambda(\delta) \leq 1-\left(1+\Psi_{x}(\delta)\right) \frac{\tilde{c}(S, n)}{G(x)} \delta^{\frac{2}{n+1}}$ for a suitable $\Psi_{x}$.

To establish the opposite inequality, we use techniques similar to the ones for the lower bound in the proof of [[53, Lemma 3].

Translate and rotate $S^{\circ}$ to a convex body $K$ such that $y$ is mapped to the origin and the outer normal is $u_{K}(0)=-e_{n}$. Let $f: \tau B_{2}^{n-1} \rightarrow \mathbb{R}$ be a parametrization of the boundary of $K$ near the origin. Let $\varepsilon>0$ and choose $\eta>0$ such that

$$
\frac{1}{n}(1-\eta)^{n-1}-\frac{1}{n+1}(1+\eta)^{n-1} \geq \frac{1}{n(n+1)} \frac{1}{(1+\varepsilon)^{\frac{n+1}{2}}} .
$$

Then there exists $\Delta_{0}>0$ such that for every $0 \leq \Delta \leq \Delta_{0}$

$$
(1-\eta) \sqrt{2 \Delta} \mathcal{E} \subseteq\left\{z \in \tau B_{2}^{n-1}: f(z) \leq \Delta\right\} \subseteq(1+\eta) \sqrt{2 \Delta} \mathcal{E}
$$

where $\mathcal{E}=\left\{z \in \mathbb{R}^{n-1}:\langle H f(0) z, z\rangle \leq 1\right\}$ is the indicatrix of Dupin (see, e.g., 34, [43]). We conclude that for every $\zeta \in \mathbb{R}^{n-1}$

$$
\operatorname{conv}\left[K, \zeta-\Delta e_{n}\right] \backslash K \supseteq \operatorname{conv}\left[\Delta e_{n}+(1-\eta) \sqrt{2 \Delta} \mathcal{E}, \zeta-\Delta e_{n}\right] \backslash K
$$

We compute

$$
\begin{gathered}
\left|\operatorname{conv}\left[K, \zeta-\Delta e_{n}\right] \backslash K\right|_{n} \geq\left|\operatorname{conv}\left[\Delta e_{n}+(1-\eta) \sqrt{2 \Delta} \mathcal{E}, \zeta-\Delta e_{n}\right] \backslash K\right|_{n} \\
\geq\left|\operatorname{conv}\left[\Delta e_{n}+(1-\eta) \sqrt{2 \Delta} \mathcal{E}, \zeta-\Delta e_{n}\right]\right|_{n}-\left|\left\{v \in K: v_{n} \leq \Delta\right\}\right|_{n} .
\end{gathered}
$$

We have

$$
\left|\operatorname{conv}\left[\Delta e_{n}+(1-\eta) \sqrt{2 \Delta} \mathcal{E}, \zeta-\Delta e_{n}\right]\right|_{n}=(1-\eta)^{n-1} \frac{(2 \Delta)^{\frac{n+1}{2}}}{n}|\mathcal{E}|_{n-1}
$$

and

$$
\begin{aligned}
\left|\left\{v \in K: v_{n} \leq \Delta\right\}\right|_{n} & \leq \int_{0}^{\Delta}\left|\left\{z \in \mathbb{R}^{n-1}: f(z) \leq t\right\}\right|_{n-1} \mathrm{~d} t \\
& \leq(1+\eta)^{n-1} \int_{0}^{\Delta}(2 t)^{\frac{n-1}{2}}|\mathcal{E}|_{n-1} \mathrm{~d} t=(1+\eta)^{n-1} \frac{(2 \Delta)^{\frac{n+1}{2}}}{n+1}|\mathcal{E}|_{n-1} .
\end{aligned}
$$

Therefore,

$$
\left|\operatorname{conv}\left[K, \zeta-\Delta e_{n}\right] \backslash K\right|_{n} \geq(2 \Delta)^{\frac{n+1}{2}}|\mathcal{E}|_{n-1}\left(\frac{(1-\eta)^{n-1}}{n}-\frac{(1+\eta)^{n-1}}{n+1}\right) \geq \frac{(2 \Delta)^{\frac{n+1}{2}}}{n(n+1)} \frac{|\mathcal{E}|_{n-1}}{(1+\varepsilon)^{\frac{n+1}{2}}}
$$


Suppose that $\delta_{0}>0$ is sufficiently small such that for every $0 \leq \delta \leq \delta_{0}$ we have

$$
\frac{\left(2 \Delta_{0}\right)^{\frac{n+1}{2}}}{n(n+1)} \frac{|\mathcal{E}|_{n-1}}{(1+\varepsilon)^{\frac{n+1}{2}}} \geq \delta|K|_{n} .
$$

Hence, if $0 \leq \delta \leq \delta_{0}$ and $\Delta \geq 0$ is chosen such that

$$
\left|\operatorname{conv}\left[K, \zeta-\Delta e_{n}\right] \backslash K\right|_{n}=\delta|K|_{n}
$$

it follows that $\Delta \leq \Delta_{0}$ and we conclude that

$$
\delta|K| \geq \frac{(2 \Delta)^{\frac{n+1}{2}}}{n(n+1)} \frac{|\mathcal{E}|_{n-1}}{(1+\varepsilon)^{\frac{n+1}{2}}}
$$

or, equivalently

$$
\Delta \leq(1+\varepsilon) \frac{1}{2}\left[n(n+1) \frac{|K|_{n}}{\left|B_{2}^{n-1}\right|_{n-1}}\right]^{\frac{2}{n+1}} \kappa_{K}(0)^{\frac{1}{n+1}} \delta^{\frac{2}{n+1}}
$$

Hence,

$$
K^{\delta} \subseteq\left\{\xi \in \mathbb{R}^{n}: \xi_{n} \geq-(1+\varepsilon) \frac{1}{2}\left[n(n+1) \frac{|K|_{n}}{\left|B_{2}^{n-1}\right|_{n-1}}\right]^{\frac{2}{n+1}} \kappa_{K}(0)^{\frac{1}{n+1}} \delta^{\frac{2}{n+1}}\right\} .
$$

Thus,

$$
\left(S^{\circ}\right)^{\delta} \subseteq\left\{v \in \mathbb{R}^{n}:\langle v-y, u(y)\rangle \leq(1+\varepsilon) n^{\frac{2}{n+1}} c\left(S^{\circ}, n\right) \kappa(y)^{\frac{1}{n+1}} \delta^{\frac{2}{n+1}}\right\} .
$$

Let $x \in \partial S$ be the unique point such that $\langle x, y\rangle=1$. It follows that for $0 \leq \delta \leq \delta_{0}$

$$
\begin{aligned}
\lambda(\delta) & \geq\left(1+(1+\varepsilon) n^{\frac{2}{n+1}} c\left(S^{\circ}, n\right) \frac{\kappa(y)^{\frac{1}{n+1}}}{\langle y, u(y)\rangle} \delta^{\frac{2}{n+1}}\right)^{-1} \geq 1-(1+\varepsilon) n^{\frac{2}{n+1}} c\left(S^{\circ}, n\right) \frac{\kappa(y)^{\frac{1}{n+1}}}{\langle y, u(y)\rangle} \delta^{\frac{2}{n+1}} \\
& =1-(1+\varepsilon) n^{\frac{2}{n+1}} c\left(S^{\circ}, n\right) \frac{\langle x, u(x)\rangle}{\kappa(x)^{\frac{1}{n+1}}} \delta^{\frac{2}{n+1}}=1-(1+\varepsilon) \frac{\tilde{c}(S, n)}{G(x)} \delta^{\frac{2}{n+1}} .
\end{aligned}
$$

This proves that we may choose a function $\Psi_{x}$ such that $\lim _{\delta \rightarrow 0} \Psi_{x}(\delta)=0$ and

$$
\left(1-\left(1+\Psi_{x}(\delta)\right) \frac{\tilde{c}(S, n)}{G(x)}\right) x \in \partial\left[\langle S\rangle^{\delta}\right] .
$$

\subsection{The $C_{+}^{2}$-Case}

Proposition 4.5 Suppose that $S$ has $C_{+}^{2}$-boundary. Then

$$
\liminf _{\delta \rightarrow 0} \frac{\mathrm{d}_{S}(\delta)-1}{\delta^{\frac{2}{n+1}}} \geq G_{\max }-G_{\min } .
$$

Proof. For $x \in \partial S$ apply Lemma 4.2 and put $\delta^{\prime \prime}=\tilde{c}(S, n)^{\frac{n+1}{2}} \delta^{\prime}$, i.e.,

$$
\left(1-\left(1+\Omega_{x}\left(\delta^{\prime \prime}\right)\right) G(x)^{-1} \delta^{\prime \prime \frac{2}{n+1}}\right) x \in \partial\langle S\rangle^{\delta^{\prime}},
$$


where $\Omega_{x}\left(\delta^{\prime \prime}\right)=\Psi_{x}\left(\delta^{\prime}\right)$. Let $x_{0} \in \partial S$ be such that $G_{\min }=\min _{x \in \partial S} G(x)=G\left(x_{0}\right)$ and let $x_{1} \in \partial S$ be such that $G_{\max }=\max _{x \in \partial S} G(x)=G\left(x_{1}\right)$. Let $a \geq 1$ be such that $\frac{1}{a} S_{\delta} \subseteq\left[\left(S^{\circ}\right)^{\delta^{\prime}}\right]^{\circ} \subseteq a S_{\delta}$. We conclude that

$$
a \geq \frac{1-\left(1+\Phi_{x_{0}}(\delta)\right) G_{\min } \delta^{\frac{2}{n+1}}}{1-\left(1+\Omega_{x_{0}}\left(\delta^{\prime \prime}\right)\right) G_{\min }^{-1} \delta^{\prime \prime \frac{2}{n+1}}}
$$

and

$$
a \geq \frac{1-\left(1+\Omega_{x_{1}}\left(\delta^{\prime \prime}\right)\right) G_{\max }^{-1} \delta^{\prime \prime \frac{2}{n+1}}}{1-\left(1+\Phi_{x_{1}}(\delta)\right) G_{\max } \delta^{\frac{2}{n+1}}}
$$

The assumption that $\delta^{\prime \prime} \geq\left(G_{\min } G_{\max }\right)^{\frac{n+1}{2}} \delta$ and inequality 4.2 lead to

$$
\liminf _{\delta>0} \frac{a-1}{\delta^{\frac{2}{n+1}}} \geq G_{\max }-G_{\min } .
$$

The assumption $\delta^{\prime \prime} \leq\left(G_{\min } G_{\max }\right)^{\frac{n+1}{2}} \delta$ and 4.3 lead to

$$
\liminf _{\delta>0} \frac{a-1}{\delta^{\frac{2}{n+1}}} \geq G_{\max }-G_{\min }
$$

as well. We conclude that

$$
\liminf _{\delta \rightarrow 0} \frac{\mathrm{d}_{S}(\delta)-1}{\delta^{\frac{2}{n+1}}} \geq G_{\max }-G_{\min }
$$

\subsection{The $B_{p}^{n}$-Case}

In this section we prove the following.

Theorem 4.6 For every $n \geq 2$ and $2<p<\infty$ we have

$$
\lim _{\delta \rightarrow 0} \frac{\mathrm{d}_{p}(\delta)-1}{\delta^{\frac{2}{n+1}}}=G\left(\left(n^{-1 / p}, \ldots, n^{-1 / p}\right)\right)=G_{\max }=G_{\max }-G_{\min } .
$$

Remark 4.1 By Lemma 4.9 below, the value of $G_{\max }$ is given by

$$
G\left(\left(n^{-1 / p}, \ldots, n^{-1 / p}\right)\right)=c\left(B_{p}^{n}, n\right)(p-1)^{\frac{n-1}{n+1}} n^{\frac{2 n+p}{(n+1) p}} .
$$

Furthermore, we give a lower bound for the case $1<p<2$. First, we need some preparatory definitions and lemmas.

Definition 4.1 Let $1 \leq p \leq \infty$. The cap $C_{p}^{n}(\Delta)$ of $B_{p}^{n}$ of height $0 \leq \Delta \leq 1$ is defined by

$$
C_{p}^{n}(\Delta)=\left\{x \in B_{p}^{n}: x_{1} \geq 1-\Delta\right\}
$$

The hat $H_{p}^{n}(\Delta)$ of height $\Delta \geq 0$ is defined by

$$
H_{p}^{n}(\Delta)=\operatorname{conv}\left[B_{p}^{n},(1+\Delta) e_{1}\right] \backslash B_{p}^{n} .
$$


Lemma 4.7 Let $1 \leq p<\infty$ and $0 \leq \Delta \leq 1$. Then

$$
\left|C_{p}^{n}(\Delta)\right|_{n}=\frac{1}{n-1+p}\left|B_{p}^{n-1}\right|_{n-1}(p \Delta)^{\frac{n-1+p}{p}}\left(1-\phi_{p}^{n}(\Delta)\right),
$$

where $\phi_{p}^{n}$ is a function with $\lim _{\Delta \rightarrow 0} \phi_{p}^{n}(\Delta)=0$.

Proof. By Taylor's theorem, for every $0<s<1$ there is $0<\sigma(s)<s$ such that $(1-s)^{p}=$ $1-p(1-\sigma(s))^{p-1} s$. This yields

$$
\int_{0}^{\Delta}\left(1-(1-s)^{p}\right)^{\frac{n-1}{p}} \mathrm{~d} s=\int_{0}^{\Delta}\left(p(1-\sigma(s))^{p-1} s\right)^{\frac{n-1}{p}} \mathrm{~d} s \leq \int_{0}^{\Delta}(p s)^{\frac{n-1}{p}} \mathrm{~d} s=\frac{1}{n-1+p}(p \Delta)^{\frac{n-1+p}{p}}
$$

and

$$
\int_{0}^{\Delta}\left(p(1-\sigma(s))^{p-1} s\right)^{\frac{n-1}{p}} \mathrm{~d} s \geq(1-\Delta)^{\frac{p-1}{p}(n-1)} \int_{0}^{\Delta}(p s)^{\frac{n-1}{p}} \mathrm{~d} s=(1-\Delta)^{\frac{p-1}{p}(n-1)} \frac{1}{n-1+p}(p \Delta)^{\frac{n-1+p}{p}} .
$$

Hence, we obtain by Cavalieri's principle

$$
\left|C_{p}^{n}(\Delta)\right|_{n}=\frac{1}{n-1+p}\left|B_{p}^{n-1}\right|_{n-1}(p \Delta)^{\frac{n-1+p}{p}}\left(1-\phi_{p}^{n}(\Delta)\right),
$$

with $0 \leq \phi_{p}^{n}(\Delta) \leq 1-(1-\Delta)^{\frac{p-1}{p}(n-1)}$.

Lemma 4.8 Let $1<p<\infty$ and $0 \leq \Delta \leq 1$. Then

$$
\left|H_{p}^{n}(\Delta)\right|_{n}=\frac{p-1}{n(n-1+p)}\left|B_{p}^{n-1}\right|_{n-1}\left(\frac{p}{p-1} \Delta\right)^{\frac{n-1+p}{p}}\left(1+\psi_{p}^{n}(\Delta)\right),
$$

where $\psi_{p}^{n}$ is a function with $\lim _{\Delta \rightarrow 0} \psi_{p}^{n}(\Delta)=0$.

Proof. Put $f(t)=\left(1-t^{p}\right)^{\frac{1}{p}},-1<t<1$. The tangential function $T_{t_{0}}(t)$ of $f$ at $t_{0}$ is given by

$$
T_{t_{0}}(t)=f\left(t_{0}\right)+f^{\prime}\left(t_{0}\right)\left(t-t_{0}\right)=\left(1-t_{0}^{p}\right)^{\frac{1}{p}-1}\left(1-t_{0}^{p-1} t\right) .
$$

If $T_{t_{0}}(1+\Delta)=0$, then $t_{0}=\frac{1}{(1+\Delta)^{\frac{1}{p-1}}}$. It follows that the boundary of the cap of $B_{p}^{n}$ touches $B_{p}^{n}$ at height $\frac{1}{(1+\Delta)^{\frac{1}{p-1}}}$ with respect to the direction $e_{1}$. The hat volume is given by the difference of the cone volume and the corresponding cap volume,

$$
\left|H_{p}^{n}(\Delta)\right|_{n}=\frac{1}{n}\left(1+\Delta-\frac{1}{(1+\Delta)^{\frac{1}{p-1}}}\right)\left(1-\frac{1}{(1+\Delta)^{\frac{p}{p-1}}}\right)^{\frac{n-1}{p}}\left|B_{p}^{n-1}\right|_{n-1}-\left|C_{p}^{n}\left(1-\frac{1}{(1+\Delta)^{\frac{1}{p-1}}}\right)\right|_{n} .
$$

Hence,

$$
\begin{aligned}
\lim _{\Delta \rightarrow 0} \frac{1}{\Delta^{\frac{n-1+p}{p}}}\left|H_{p}^{n}(\Delta)\right|_{n} & =\frac{1}{n}\left(\frac{p}{p-1}\right)^{\frac{n-1+p}{p}}\left|B_{p}^{n-1}\right|_{n-1}-\frac{1}{n-1+p}\left(\frac{p}{p-1}\right)^{\frac{n-1+p}{p}}\left|B_{p}^{n-1}\right|_{n-1} \\
& =\frac{p-1}{n(n-1+p)}\left(\frac{p}{p-1}\right)^{\frac{n-1+p}{p}}\left|B_{p}^{n-1}\right|_{n-1} .
\end{aligned}
$$


The following formulas for the Gauss curvature and the normal directions of $B_{p}^{n}$ can be found in e.g. [20, 41].

Lemma 4.9 The Gauss curvature at $x \in \partial B_{p}^{n}$ is given by

$$
\kappa(x)=(p-1)^{n-1} \frac{\prod_{i=1}^{n}\left|x_{i}\right|^{p-2}}{\left(\sum_{i=1}^{n}\left|x_{i}\right|^{2 p-2}\right)^{\frac{n+1}{2}}}
$$

and the normal is given by

$$
u(x)=\frac{1}{\left(\sum_{i=1}^{n}\left|x_{i}\right|^{2 p-2}\right)^{\frac{1}{2}}}\left(\operatorname{sign}\left(x_{i}\right)\left|x_{i}\right|^{p-1}\right)_{i=1}^{n},
$$

if $2 \leq p<\infty$ or if $1<p<2$ and all components of $x$ are not equal to zero.

Lemma 4.10 Let $1<p<\infty$.

1. There are functions $\Phi_{1}, \Phi_{2}$, depending only on $n$ and $p$, such that $\lim _{\delta \rightarrow 0} \Phi_{1}(\delta)=0, \lim _{\delta \rightarrow 0} \Phi_{2}(\delta)=0$ and

$$
\begin{gathered}
\pm\left(1-\left(1+\Phi_{1}(\delta)\right) c_{1} \delta^{\frac{p}{n-1+p}}\right) e_{i} \in \partial\left(B_{p}^{n}\right)_{\delta}, \quad 1 \leq i \leq n, \\
\left(1-\left(1+\Phi_{2}(\delta)\right) c_{2} \delta^{\frac{2}{n+1}}\right) \frac{1}{\sqrt[p]{n}} \sum_{i=1}^{n} \pm e_{i} \in \partial\left(B_{p}^{n}\right)_{\delta}
\end{gathered}
$$

where

$$
c_{1}=\frac{(n-1+p)^{\frac{p}{n-1+p}}}{p}\left(\frac{\left|B_{p}^{n}\right|_{n}}{\left|B_{p}^{n-1}\right|_{n-1}}\right)^{\frac{p}{n-1+p}}, \quad c_{2}=G_{B_{p}^{n}}\left(\left(n^{-1 / p}, \ldots, n^{-1 / p}\right)\right) .
$$

2. There are functions $\Psi_{1}, \Psi_{2}$, depending only on $n$ and $p$, such that $\lim _{\delta \rightarrow 0} \Psi_{1}(\delta)=0, \lim _{\delta \rightarrow 0} \Psi_{2}(\delta)=0$ and

$$
\begin{aligned}
\pm\left(1-\left(1+\Psi_{1}(\delta)\right) c_{3} \delta^{\frac{p}{n-1+p}}\right) e_{i} \in \partial\left\langle B_{p^{\prime}}^{n}\right\rangle^{\delta}, \quad 1 \leq i \leq n, \\
\left(1-\left(1+\Psi_{2}(\delta)\right) c_{4} \delta^{\frac{2}{n+1}}\right) \frac{1}{\sqrt[p]{n}} \sum_{i=1}^{n} \pm e_{i} \in \partial\left\langle B_{p^{\prime}}^{n}\right\rangle^{\delta},
\end{aligned}
$$

where $\frac{1}{p}+\frac{1}{p^{\prime}}=1$ and

$$
c_{3}=\left[\frac{n\left(n-1+p^{\prime}\right)}{p^{\prime}-1} \cdot \frac{\left|B_{p^{\prime}}^{n}\right|_{n}}{\left|B_{p^{\prime}}^{n-1}\right|_{n-1}}\right]^{\frac{p^{\prime}}{n-1+p^{\prime}}} \cdot \frac{p^{\prime}-1}{p^{\prime}}, \quad c_{4}=n^{\frac{2}{n+1}} G_{B_{p^{\prime}}^{n}}\left(\left(n^{-1 / p^{\prime}}, \ldots, n^{-1 / p^{\prime}}\right)\right) .
$$

Proof. Part 1. is an immediate consequence of Lemmas 4.7 and 4.3 For Part 2., note that by Lemma 4.7

$$
\pm\left(1+\left(1+\psi_{1}(\delta)\right) c_{3} \delta^{\frac{p}{n-1+p}}\right) e_{i} \in \partial\left[\left(B_{p}^{n}\right)^{\delta}\right] .
$$


By Lemma 4.4

$$
\left(1+\left(1+\psi_{2}(\delta)\right) c_{4} \delta^{\frac{2}{n+1}}\right) \frac{1}{\sqrt[p]{n}} \sum_{i=1}^{n} \pm e_{i} \in \partial\left[\left(B_{p}^{n}\right)^{\delta}\right] \quad,
$$

for some functions $\psi_{1}, \psi_{2}$ with $\lim _{\delta \rightarrow 0} \psi_{1}(\delta)=0$ and $\lim _{\delta \rightarrow 0} \psi_{2}(\delta)=0$. Furthermore, note that by symmetry $\pm e_{i}$ is an outer normal of $\left(B_{p}^{n}\right)^{\delta}$ at $\pm\left(1+\left(1+\psi_{1}(\delta)\right) c_{3} \delta^{\frac{p}{n-1+p}}\right) e_{i}$ and $\left(n^{-1 / 2}, \ldots, n^{-1 / 2}\right)$ is an outer normal of $\left(B_{p}^{n}\right)^{\delta}$ at $\left(1+\left(1+\psi_{2}(\delta)\right) c_{4} \delta^{\frac{2}{n+1}}\right) \frac{1}{\sqrt[p]{n}} \sum_{i=1}^{n} \pm e_{i}$. Similar to the proof of Part 2 . of Lemma 4.2 , one can show that

$$
\pm\left(1+\left(1+\psi_{1}(\delta)\right) c_{3} \delta^{\frac{p}{n-1+p}}\right)^{-1} e_{i} \in\left\langle B_{p^{\prime}}^{n}\right\rangle^{\delta}
$$

and

$$
\left(1+\left(1+\psi_{2}(\delta)\right) c_{4} \delta^{\frac{2}{n+1}}\right)^{-1} \frac{1}{\sqrt[p^{\prime}]{n}} \sum_{i=1}^{n} \pm e_{i} \in\left\langle B_{p^{\prime}}^{n}\right\rangle^{\delta} .
$$

From this, we conclude.

Proposition 4.11 Let $2 \leq p<\infty$ and let $c_{2}$ be the constant of Lemma 4.10. Then we have

$$
\liminf _{\delta \rightarrow 0} \frac{\mathrm{d}_{p}(\delta)-1}{\delta^{\frac{2}{n+1}}} \geq c_{2}=G_{\max }=G\left(\left(n^{-1 / p}, \ldots, n^{-1 / p}\right)\right)
$$

Proof. Fix $\delta^{\prime} \geq 0$ and let $a_{\delta} \geq 1$ be such that

$$
\frac{1}{a_{\delta}}\left(B_{p}^{n}\right)_{\delta} \subseteq\left\langle B_{p}^{n}\right\rangle^{\delta^{\prime}} \subseteq a_{\delta}\left(B_{p}^{n}\right)_{\delta}
$$

From Lemma 4.10 we deduce that

$$
a_{\delta} \geq \max \left[\frac{1-c_{4} \delta^{\prime \frac{2}{n+1}}\left(1+\Psi_{4}\left(\delta^{\prime}\right)\right)}{1-c_{2} \delta^{\frac{2}{n+1}}\left(1+\Phi_{2}(\delta)\right)}, \frac{1-c_{1} \delta^{\frac{p}{n-1+p}}\left(1+\Phi_{1}(\delta)\right)}{1-c_{3} \delta^{\frac{p^{\prime}}{n-1+p^{\prime}}}\left(1+\Psi_{3}\left(\delta^{\prime}\right)\right)}\right]
$$

Since $\frac{p^{\prime}}{n-1+p^{\prime}}<\frac{2}{n+1}<\frac{p}{n-1+p}$, we may choose a fixed constant $1<\alpha<\frac{2}{n+1} \cdot \frac{n-1+p^{\prime}}{p^{\prime}}$. As $\alpha \frac{p^{\prime}}{n-1+p^{\prime}}<$ $\frac{2}{n+1}$, we get for $\delta^{\prime} \geq \delta^{\alpha}$ that

$$
\begin{aligned}
a_{\delta} & \geq \frac{1-c_{1} \delta^{\frac{p}{n-1+p}}\left(1+s_{1}(\delta)\right)}{1-c_{3} \delta^{\alpha \frac{p^{\prime}}{n-1+p^{\prime}}}\left(1+s_{3}\left(\delta^{\prime}\right)\right)}=1+c_{3} \delta^{\alpha \frac{p^{\prime}}{n-1+p^{\prime}}}\left(1+o_{\delta}(1)+o_{\delta^{\prime}}(1)\right) \\
& \geq 1+c_{2} \delta^{\frac{2}{n+1}}\left(1+o_{\delta}(1)+o_{\delta^{\prime}}(1)\right) .
\end{aligned}
$$

If $\delta^{\prime} \leq \delta^{\alpha}$ it follows that

$$
a_{\delta} \geq \frac{1-c_{4} \delta^{\alpha \frac{2}{n+1}}\left(1+s_{4}\left(\delta^{\prime}\right)\right)}{1-c_{2} \delta^{\frac{2}{n+1}}\left(1+s_{2}(\delta)\right)}=1+c_{2} \delta^{\frac{2}{n+1}}\left(1+o_{\delta}(1)+o_{\delta^{\prime}}(1)\right)
$$

We may assume without loss of generality that $\delta^{\prime}$ tends to zero as $\delta$ tends to zero and hence,

$$
\lim _{\delta \rightarrow 0} \frac{a_{\delta}-1}{\delta^{\frac{2}{n+1}}} \geq c_{2}
$$


The last step is to prove that $G_{\max }=G\left(\left(n^{-1 / p}, \ldots, n^{-1 / p}\right)\right)$. By Lemma 4.9 .

$$
\frac{\kappa(x)^{\frac{1}{n+1}}}{\langle x, u(x)\rangle}=(p-1)^{\frac{n-1}{n+1}} \prod_{i=1}^{n}\left|x_{i}\right|^{\frac{p-2}{n+1}}
$$

The arithmetic geometric means inequality yields

$$
\prod_{i=1}^{n}\left|x_{i}\right|^{\frac{p-2}{n+1}}=\left(\sqrt[n]{\prod_{i=1}^{n}\left|x_{i}\right|^{p}}\right)^{\frac{n}{n+1} \cdot \frac{p-2}{p}} \leq\left(\frac{1}{n}\left(\sum_{i=1}^{n}|x|_{i}^{p}\right)\right)^{\frac{n}{n+1} \cdot \frac{p-2}{p}}=n^{-\frac{n}{n+1} \cdot \frac{p-2}{p}}
$$

, with equality if and only if all $\left|x_{i}\right|$ are equal to $n^{-1 / p}$.

Proposition 4.12 Let $1<p<2$ and let $c_{1}$ be the constant from Lemma 4.10. We have

$$
\liminf _{\delta \rightarrow 0} \frac{\mathrm{d}_{p}(\delta)-1}{\delta^{\frac{p}{n-1+p}}} \geq c_{1}=\frac{(n-1+p)^{\frac{p}{n-1+p}}}{p}\left(\frac{\left|B_{p}^{n}\right|_{n}}{\left|B_{p}^{n-1}\right|_{n-1}}\right)^{\frac{p}{n-1+p}}
$$

Proof. Similarly as in the previous proposition one argues that

$$
a_{\delta} \geq \max \left[\frac{1-c_{2} \delta^{\frac{2}{n+1}}\left(1+o_{\delta}(1)\right)}{1-c_{4} \delta^{\frac{2}{n+1}}\left(1+o_{\delta^{\prime}}(1)\right)}, \frac{1-c_{3} \delta^{\frac{p^{\prime}}{n-1+p^{\prime}}}}{1-c_{1} \delta^{\frac{p}{n-1+p}}}\right] .
$$

Note that we have $\frac{p}{n-1+p}<\frac{2}{n+1}<\frac{p^{\prime}}{n-1+p^{\prime}}$. We fix an $\alpha>0$ with

$$
\frac{p}{n-1+p} \cdot \frac{n-1+p^{\prime}}{p^{\prime}}<\alpha<\frac{p}{n-1+p} \cdot \frac{n+1}{2}
$$

and we conclude as in the previous proof by considering the two cases $\delta^{\prime} \leq \delta^{\alpha}$ and $\delta^{\prime} \geq \delta^{\alpha}$.

\section{Acknowledgement}

The first author likes to thank the Department of Mathematics at Case Western Reserve University, Cleveland, for their hospitality during his research stay in 2015/2016. Both authors want to thank the Mathematical Science Research Institute, Berkley. It was during a stay there when the paper was completed.

\section{References}

[1] B. Andrews, The affine curve-lengthening flow, J. Reine Angew. Math. 506 (1999), 43-83.

[2] S. Artstein-Avidan, B. Klartag, C. Schütt, and E. M. Werner, Functional affine-isoperimetry and an inverse logarithmic Sobolev inequality, J. Funct. Anal. 262 (2012), no. 9, 4181-4204.

[3] I. Bárány, Random polytopes in smooth convex bodies, Mathematika 39 (1992), 81-92; Corrigendum, Mathematika 51 (2004), 31. 
[4] I. Bárány and D. G. Larman, Convex bodies, economic cap coverings, random polytopes, Mathematika 35 (1988), no. 2, 274-291.

[5] F. Besau, M. Ludwig and E.M. Werner, Weighted floating bodies and polytopal approximation, to appear in Transactions of the AMS.

[6] F. Besau and E.M. Werner, The spherical convex floating body, Adv. Math. 301 (2016), 867-901.

[7] F. Besau and E.M. Werner, The floating body in real space forms, to appear in Journal of Differential Geometry.

[8] K. Böröczky, Jr., Approximation of general smooth convex bodies, Adv. Math. 153 (2000), 325341.

[9] K. Böröczky, Jr., Polytopal approximation bounding the number of k-faces., J. Approx. Theory 102 (2000), 263-285.

[10] K. Böröczky Jr., E. Lutwak, D. Yang, G. Zhang, The Logarithmic Minkowski Problem, Journal of the AMS 26 (2013), 831-852.

[11] A. Colesanti, G. Livshyts, A. Marsiglietti, On the stability of Brunn-Minkowski type inequalities, https://arxiv.org/pdf/1606.06586.pdf.

[12] C. Dupin, Applications de géométrie et de méchanique, Paris, 1822.

[13] R.J. Gardner, Geometric tomography, Second, Encyclopedia of Mathematics and its Applications, vol. 58, Cambridge University Press, Cambridge (2006), MR2251886, Zbl 1102.52002.

[14] P.M. Gruber, Convex and discrete geometry (Grundlehren der Mathematischen Wissenschaften 336). Springer, Berlin (2007).

[15] C. Haberl and F. Schuster, General $L_{p}$ affine isoperimetric inequalities, J. Differential Geom. 83 (2009), no. 1, 1-26.

[16] C. Haberl and L. Parapatits, The centro-affine Hadwiger theorem, Journal of the AMS 27 (2014), no. $3,685-705$.

[17] Y. Huang, E. Lutwak, D. Yang, G. Zhang, Geometric measures in the dual Brunn-Minkowski theory and their associated Minkowski problems, Acta Math. 216 (2016), 325-388.

[18] D. Hug, Curvature relations and affine surface area for a general convex body and its polar, Results in Math. 29 (1996), 233-248.

[19] M. N. Ivaki and A. Stancu, Volume preserving centro-affine normal flows, Comm. Anal. Geom. 21 (2013), no. 3, 671-685.

[20] A. Koldobsky, D. Ryabogin, A. Zvavitch, Projections of convex bodies and the Fourier transform, Israel J. Math., 139 (2004), 361-380.

[21] K. Leichtweiß, Über eine Formel Blaschkes zur Affinoberfläche konvexer Körper, Studia Sci. Math. Hungar. 21 (1986), 453-474.

[22] K. Leichtweiß, Zur Affinoberfläche konvexer Körper, Manuscripta Math. 56 (1986), no. 4, 429-464.

[23] M. Ludwig, Asymptotic approximation of smooth convex bodies by general polytopes, Mathematika 46 (1999), 103-125. 
[24] M. Ludwig and M. Reitzner, A characterization of affine surface area, Adv. Math. 147 (1999), $138-172$.

[25] M. Ludwig and M. Reitzner, A classification of SL( $n$ ) invariant valuations, Ann. of Math. (2) 172 (2010), 1219-1267.

[26] E. Lutwak, Extended affine surface area, Adv. Math. 85 (1991), 39-68.

[27] E. Lutwak and V. Oliker, On the regularity of solutions to a generalization of the Minkowski problem, J. Differential Geom. 41 (1995), no. 1, 227-246.

[28] E. Lutwak, The Brunn-Minkowski-Firey theory. II: Affine and geominimal surface areas, Adv. Math. 118 (1996), 244-294.

[29] M. Meyer, S. Reisner, A geometric property of the boundary of symmetric convex bodies and convexity of flotation surfaces, Geom. Ded., 39 (1991), 327-337.

[30] M. Meyer, E.M. Werner, On the p-affine surface area, Adv. Math. 152 (2000), 288-313.

[31] A. Naor, The surface measure and cone measure on the sphere of $\ell_{p}^{n}$, Transactions of the AMS, 359 (2007), 1045-1079.

[32] G. Paouris and E.M. Werner, Relative entropy of cone measures and $L_{p}$ centroid bodies, Proc. Lond. Math. Soc. (3) 104 (2012), no. 2, 253-286.

[33] C. Petty, Affine isoperimetric problems, Ann. New York Acad. Sci., 440 (1985), 113-127.

[34] S. Reisner, C. Schütt, E.M. Werner, A note on Mahler's conjecture, Int. Math. Research Not., 1 (2012), 1-16 .

[35] Random points on the boundary of smooth convex bodies, Transactions of the AMS 354 (2002), 2243-2278.

[36] M. Reitzner, The combinatorial structure of random polytopes, Adv. Math. 191 (2005), no. 1, $178-208$.

[37] M. Reitzner, Random polytopes, New Perspectives in Stochastic Geometry, Oxford Univ. Press, Oxford, 2010, 45-76.

[38] R. Schneider, Convex Bodies: The Brunn-Minkowski Theory, Cambridge University Press, Cambridge (2013).

[39] C. Schütt, The convex floating body and polyhedral approximation, Israel J. Math., 73 (1991), 65-77.

[40] C. Schütt, Random polytopes and affine surface area, Math. Nachr. 170 (1994), 227-249.

[41] C. Schütt, E.M. Werner, The convex floating body, Math. Scand. 66 (1990), 275-290.

[42] C. Schütt, E.M. Werner, Homothetic floating bodiees, Geom. Ded. 3 (1994), 335-348.

[43] C. Schütt and E. M. Werner, Polytopes with vertices chosen randomly from the boundary of a convex body, Geometric aspects of functional analysis, Lecture Notes in Math., vol. 1807, Springer, Berlin, 2003, pp. 241-422.

[44] C. Schütt and E. M. Werner, Surface bodies and p-affine surface area, Adv. Math. 187 (2004), no. $1,98-145$. 
[45] A. Stancu, The discrete planar $L_{0}$-Minkowski problem, Adv. Math. 167 (2002), no. 1, 160-174.

[46] A. Stancu, On the number of solutions to the discrete two-dimensional $L_{0}$-Minkowski problem, Adv. Math. 180 (2003), no. 1, 290-323.

[47] A. Stancu, Two volume product inequalities and their applications, Canadian Math. Bulletin, 52 (2009), 464-472.

[48] N. S. Trudinger and Xu-Jia Wang, The Bernstein problem for affine maximal hypersurfaces, Invent. Math. 140 (2000), 399-422.

[49] N. S. Trudinger and Xu-Jia Wang, Affine complete locally convex hypersurfaces. Invent. Math. 150 (2002), 45-60.

[50] N. S. Trudinger and Xu-Jia Wang, The Affine Plateau Problem. Jornal of the AMS 18 (2005), no.2, 253-289.

[51] N.S. Trudinger, X. Wang, Boundary regularity for the Monge-Ampere and affine maximal surface equations, Ann. of Math. 167 (2008), 993-1028.

[52] R.J. Webster, Convexity, Oxford Science Publications, 8th edition, (1994).

[53] E.M. Werner, Illumination bodies and affine surface area, Stud. Math. 110 (1994), no. 3, 257-269-

[54] E.M. Werner, The illumination bodies of a simplex, J. of Disc. and Comp. Geometry 15 (1996), $297-306$.

[55] E.M. Werner, Floating bodies and illumination bodies, Proceedings of the conference "Integral Geometry And Convexity" Wuhan 2004, World Scientific, Singapore (2006).

[56] E.M. Werner, Rényi divergence and $L_{p}$-affine surface area for convex bodies, Adv. Math. 230 (2012), no. 3, 1040-1059.

[57] E.M. Werner, D. Ye, On the homothety conjecture, Indiana Univ. Math. J. 60 (2011), no. 1, 1-20.

Olaf Mordhorst

Institut für diskrete Mathematik und Geometrie

Technische Universität Wien

1040 Wien, Austria

olaf.mordhorst@tuwien.ac.at

Elisabeth M. Werner

Department of Mathematics

Case Western Reserve University

Cleveland, Ohio 44106, U. S. A.

elisabeth.werner@case .edu
Université de Lille 1

UFR de Mathématique

59655 Villeneuve d'Ascq, France 